\title{
Conventional and Nanotechnology Based Approaches to Combat Chronic Obstructive Pulmonary Disease: Implications for Chronic Airway Diseases
}

This article was published in the following Dove Press journal:

International Journal of Nanomedicine

\author{
Mehak Passi ${ }^{1, *}$ \\ Sadia Shahid $\mathbb{D D}^{2, *}$ \\ Sankarakuttalam Chockalingam ${ }^{2}$ \\ Isaac Kirubakaran Sundar (iD ${ }^{3}$ \\ Gopinath Packirisamy (D) ${ }^{1,2}$ \\ 'Nanobiotechnology Laboratory, Centre \\ for Nanotechnology, Indian Institute of \\ Technology Roorkee, Roorkee 247667, \\ Uttarakhand, India; ${ }^{2}$ Department of \\ Biotechnology, Indian Institute of \\ Technology Roorkee, Roorkee 247667, \\ Uttarakhand, India; ${ }^{3}$ Department of \\ Environmental Medicine, University of \\ Rochester Medical Center, Rochester, \\ NY 14623, USA
}

*These authors contributed equally to this work
Correspondence: Isaac Kirubakaran Sundar; Gopinath Packirisamy

Tel + I-585-273-3034; +9|-|332-285650

Email isaac_sundar@urmc.rochester.edu; genegopi@gmail.com

\begin{abstract}
Chronic obstructive pulmonary disease (COPD) is the most prevalent obstructive lung disease worldwide characterized by decline in lung function. It is associated with airway obstruction, oxidative stress, chronic inflammation, mucus hypersecretion, and enhanced autophagy and cellular senescence. Cigarette smoke being the major risk factor, other secondary risk factors such as the exposure to air pollutants, occupational exposure to gases and fumes in developing countries, also contribute to the pathogenesis of COPD. Conventional therapeutic strategies of COPD are based on anti-oxidant and anti-inflammatory drugs. However, traditional anti-oxidant pharmacological therapies are commonly used to alleviate the impact of COPD as they have many associated repercussions such as low diffusion rate and inappropriate drug pharmacokinetics. Recent advances in nanotechnology and stem cell research have shed new light on the current treatment of chronic airway disease. This review is focused on some of the anti-oxidant therapies currently used in the treatment and management of COPD with more emphasis on the recent advances in nanotechnology-based therapeutics including stem cell and gene therapy approaches for the treatment of chronic airway disease such as COPD and asthma.
\end{abstract}

Keywords: chronic obstructive pulmonary disease, COPD, nanotechnology, anti-oxidant therapy, stem cell therapy, gene therapy

\section{Introduction}

Chronic obstructive pulmonary disease (COPD) is a disease characterized by poor airflow in the lungs for long term. According to a prediction by the World Health Organization (WHO), by the end of 2030 COPD will be the third most common cause of global deaths. Various factors that contribute to the pathogenesis of COPD such as oxidative stress, inflammatory response, and changes in biological processes cell proliferation, alternation in programmed cell death and cellular senescence. Among all the factors, oxidative stress plays a major role in the pathogenesis of COPD. Both exogenous sources such as cigarette smoke (CS) and air pollution as well as endogenous sources such as reactive oxygen species (ROS) and reactive nitrogen species (RNS) released by activated macrophages and neutrophils contribute to the increased oxidative burden in the lungs. Cigarette smoke generates $10^{4}$ harmful oxidants with respect to each puff that human inhales playing a pivotal role in the pathogenesis of COPD causing an imbalance between oxidant and 


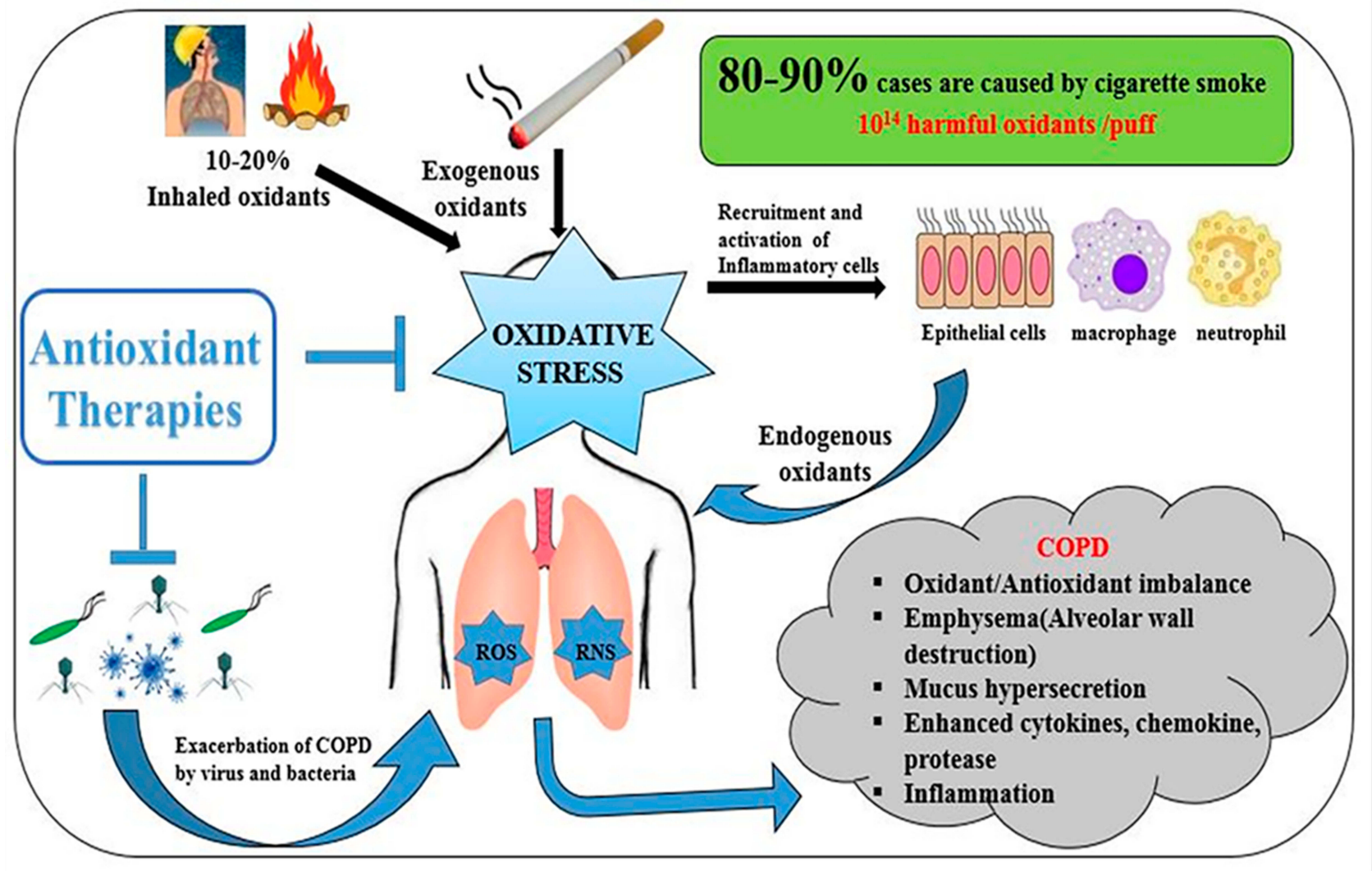

Figure I Factors that influence the pathogenesis of COPD.

antioxidant species (Figure 1). Hence, powerful therapeutic agents targeting lungs that have the potential to boost up endogenous antioxidant or alter the redox system could offer a useful therapeutic approach in reducing oxidative stress. ${ }^{1-3}$

In this review, we discuss some of the conventional antioxidant therapies along with the recent gene and stem cellbased therapies. Additionally, nanotechnology-based therapeutic approaches used in the treatment of COPD along with its advantages and disadvantages are discussed in detail.

Till now various antioxidants have been used to combat oxidative stress such as NAC (N-acetyl-L-cysteine), Nrf2 activators, enzyme mimetics, and spin traps. NAC is a good antioxidant as well as mucolytic agent but possess lower bioavailability. Nrf2 is a transcription factor that plays a role in activating Antioxidant Response Element (ARE) that is responsible for the expression of various Phase II antioxidant genes. Potential Nrf2 activators are sulforaphane, curcumin, terpenoids etc. ${ }^{4-6}$ Enzyme mimetics mimic the normal functions of various antioxidant enzymes such as superoxide dismutase, glutathione peroxidase and catalase, while spin traps convert the reactive oxygen species into a stable product so that it does not have any effect on the body. However, there are many disadvantages associated with the use of these antioxidant pharmacological strategies such as low diffusion rate and inappropriate pharmacokinetics of the drugs that result in unsatisfactory response to the treatment of chronic lung disease. Also, the genetic molecules are not easily delivered to the cells since they are mostly degraded by biological fluids. Nanotechnology offers promising opportunities for the fabrication of various nanocarriers to target COPD. These nanocarriers possess unique innate physical properties and enhance the pharmacokinetics of the loaded therapeutic molecules. Also, these nanocarriers can be conjugated with the diverse targeting ligands specific for receptors expressed in the diseased cells which can be used to target delivery minimizing the adverse effects associated with the drugs. Nanocarriers can even act as an efficient vector system for successful application in gene and stem cell-based therapies. The importance of the nanotechnology in treating chronic lung disease such as COPD is discussed in later sections. Some light has also been shed on the current gene and stem cell therapy related approaches that can be used in the treatment of COPD.

\section{Antioxidant Therapies for COPD}

Agents like Nrf2 activators, N-acetyl-L-cysteine (NAC), $\mathrm{N}$-acystelyn (NAL), and N-isobutyrylcysteine (NIC) and 
catalytic antioxidants reduce oxidative burden and inflammation in the lungs. These agents control the biosynthesis of glutathione (GSH) and activation of NF- $\mathrm{KB}$ thereby affect the redox system, chromatin remodelling machinery, binding of transcription factors to the promoters of proinflammatory genes and thus reduce oxidative stress. Other agents like spin traps, carbocysteine and porphyrins also reduce inflammation that is associated with $\mathrm{COPD} .^{7,8}$

\section{$\mathrm{N}$-Acetyl-L-Cysteine (NAC)}

COPD is a disease characterized by imbalance and modulation between oxidant and antioxidant species. Glutathione (GSH) is an antioxidant molecule that reduces oxidative stress. N-acetyl-L-cysteine (NAC) is an antioxidant drug consisting of acetyl derivative of cysteine that enhances GSH levels and affects major inflammatory pathways. ${ }^{9}$ NAC in the gut is deacetylated to provide cysteine that functions as a precursor of GSH synthesis. Dodd et al demonstrated that NAC increases the levels of endogenous glutathione (Figure 2). ${ }^{10}$ While Burgunder et al have shown that NAC reduces cystine to cysteine, which is very important as cellular GSH increases in vivo in the lungs. In addition to reducing the disulfide bonds, it modulates the redox system in the cells by directly reacting with reactive oxidants thereby influence canonical inflammatory signaling pathways. ${ }^{11}$ Rubio et al have demonstrated that NAC successfully reduces the elastaseinduced emphysema in rats. ${ }^{12}$ Recently, Cazzola et al demonstrated the antioxidant and anti-inflammatory effect of NAC in a COPD exacerbation model depicting that the NAC treatment reduces the detrimental effect induced by lipopolysaccharide (LPS). ${ }^{13}$

In addition to antioxidant properties, NAC also functions as a mucolytic agent. It disrupts the crosslinking of mucus gel affecting overall mucus secretion, viscosity and elasticity. It improves mucociliary clearance and lung function. NAC even prevents bacterial-stimulated mucin release and mucus hypersecretion by clearing the airway. ${ }^{14}$ Dodd et al demonstrated that NAC was safe when orally administered, but it possessed some risk when administrated intravenously. ${ }^{10}$

NAC has widely been used in various in vitro and in vivo studies as a thiol compound. The clinical use of NAC in COPD patients produced varied results. Besaratinia et al found that with $600 \mathrm{mg}$ oral dose of NAC for 6 months in smokers decreased various oxidative biomarkers that are associated with plasma and bronchoalveolar lavage (BAL) fluid. ${ }^{15}$ Another clinical trial showed that administration of $600 \mathrm{mg}$ NAC for 2 months effectively reduced oxidative stress in airways of COPD patients. ${ }^{16}$ Moreover, this decrease in oxidative biomarkers yield better clinical results like reduced the risk of exacerbations, enhancing the lung function in patients suffering from chronic bronchitis. ${ }^{17,18}$ In addition, NAC controls the hypoxia-inducible factor- $1 \alpha$ and activation of NF- $\mathrm{KB}$ that reduces lung allergies. ${ }^{19}$ It has been found that oral administration of NAC in severe COPD patients enhanced the quadriceps endurance, implying that NAC reduces the oxidant burden associated with COPD. ${ }^{20}$ On the other hand, a double

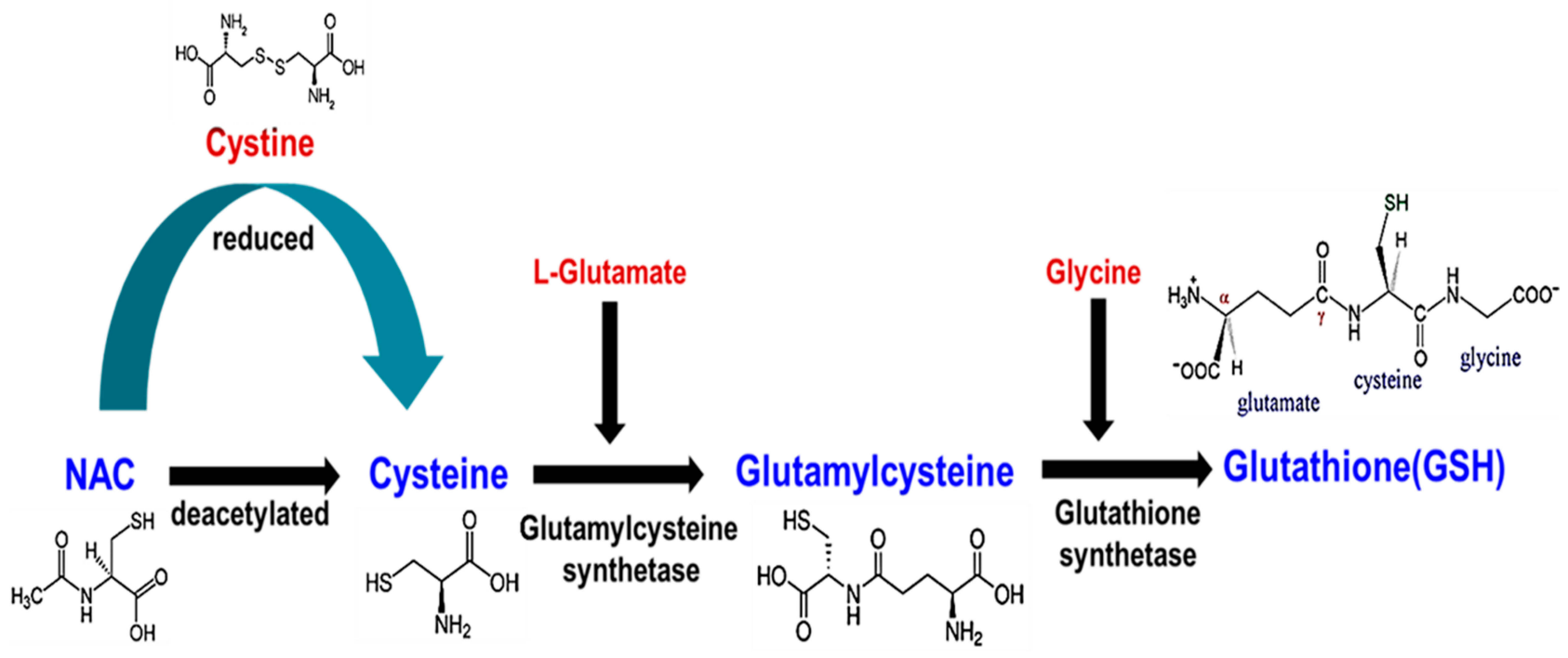

Figure 2 Mode of action of NAC and GSH synthesis. 
blind, multicenter study using NAC in patients with long term chronic cough administered by dose inhalers failed to give a positive result on mucus production and lung function. ${ }^{21}$

\section{Alternative Compounds for N-Acetyl-L- Cysteine (NAC) $\mathrm{N}$-Acystelyn (NAL) and N-Isobutyrylcysteine (NIC)} Both $\mathrm{N}$-acystelyn (NAL) and N-isobutyrylcysteine (NIC) are used as alternatives to NAC. NAL is a lysine salt of NAC. It reduces the oxidative burden, ROS- mediated inflammatory response both in vivo and in vitro by acting as both a mucolytic agent and an antioxidant thiol compound. ${ }^{22,23}$ NAL increases the levels of intracellular GSH by two folds as compared to NAC. ${ }^{24}$ Another report summarized by Elborn et al regarding the activity and tolerability of NAL in cystic fibrosis (CF) patients. They found that aerosol administration was well tolerated and it decreased mucus rigidity without causing any significant side effects. ${ }^{25}$ Therefore, NAL is more advantageous than NAC in targeting and eliminating oxidative stress in chronic lung diseases. Another advantage of NAL over other antioxidant drugs is that it can be monitored by inhalation. The second substitute to NAC is NIC. The bioavailability of NAC is less due to the fact that NAC gets hydrolyzed in living systems. NIC is a thiol compound having a higher oral bioavailability than NAC. ${ }^{26}$ When the performance of NIC was evaluated in COPD and chronic bronchitis patients, it failed to minimize exacerbation rates that limit its usefulness against NAC for therapeutic use. $^{24}$

\section{Thiol Derivatives}

There are many drawbacks associated with NAC, NAL, and NIC. So there is a need to synthesize novel thiol compounds better than already existing thiols. These thiols compounds consist of carbocysteine, erdosteine, fudosteine and procysteine that possess enhanced mucolytic properties as well as the greater ability to reduce bacterial adhesiveness.

\section{Carbocysteine}

S-carboxymethyl cysteine (S-CMC) and its lysine salt are available for oral use. Chronic bronchitis patients treated with S-CMC lysine exhibited enhanced mucociliary transport and reduced mucus viscosity. ${ }^{27,28}$ Carbocysteine has been shown to reduce human neutrophil elastase (HNE)induced mucus hypersecretion and decrease the level of ROS in NCI-H292 cell line. ${ }^{29}$ A study revealed that carbocysteine promoted cell survival and inhibited the cell damage/apoptosis caused by $\mathrm{H}_{2} \mathrm{O}_{2}$ by activating PI3KAKT pathway. ${ }^{30}$ Another study also revealed the anti- inflammatory effects of carbocysteine in human bronchial epithelial cells treated with cigarette smoke extract by altering TLR4 and IL-8 expression in vitro in 16-HBE cells. ${ }^{31}$ Moreover, carbocysteine effectively prevented the human lung carcinoma cells from $\mathrm{H}_{2} \mathrm{O}_{2}$-induced cell injury by inhibiting NF- $\mathrm{KB}$ and MAPK-ERK1/2 signalling pathways. $^{32}$ All the above-mentioned studies indicated that carbocysteine possesses both anti-oxidant and anti-inflammatory properties.

Carbocysteine even plays a pivotal role in reducing bacterial exacerbations in COPD by preventing the bacterial attachment to the cell membrane. ${ }^{33}$ In another report, it was demonstrated that carbocysteine reduced the adherence of Moraxella catarrhalis (commonly involved bacteria in COPD exacerbation) to pharyngeal epithelial cells dose-dependently as compared to control cells. ${ }^{34}$ Carbocysteine also downregulated the adhesion molecule-1 and inhibited the attachment of Streptococcus pneumoniae to human pharyngeal epithelial cells. ${ }^{35}$

\section{Erdosteine}

Erdosteine is a multifunctional drug that possess versatile properties such as, acting as a mucolytic agent and also reduces the viscosity and elastic properties of sputum. It even inhibits the attachment of bacteria to the cell surfaces by acting as an antibacterial agent. Besides, it even scavenges free radicals and ROS, it acts as an anti-inflammatory agent. ${ }^{36}$ Many clinical trials have proven the protective effect of erdosteine on COPD exacerbation by scavenging ROS. COPD patients treated with erdosteine $300 \mathrm{mg}$ twice a day for 8 months ${ }^{37}$ and $300 \mathrm{mg}$ twice for 7-10 days $^{38}$ demonstrated an improved reduction in exacerbation and hospitalization rates. Overall, it improved the health status of acute exacerbation in COPD (AECOPD) patients. ${ }^{36}$

In another study, they showed that 40-80 years old patients suffering from COPD that received $300 \mathrm{mg}$ erdosteine for 12 months show reduced COPD exacerbation, owing to its excellent anti-inflammatory and adhesive properties. ${ }^{39}$ Erdosteine treatment benefits patients suffering from repeated and severe COPD exacerbations. ${ }^{40}$ The inflammatory properties of the erdosteine were also studied by treatment with $600 \mathrm{mg}$ erdosteine a day. It significantly led to the reduction of cigarette smoke-induced ROS produced by activated macrophages and maintained the IL-6 and IL-8 cytokine levels in bronchial secretions of patients with COPD. ${ }^{41}$ Another study demonstrated a reduction in inflammatory eicosanoids in the blood of COPD patients. ${ }^{42}$ 


\section{Fudosteine}

Fudosteine is a propionic acid that possess both mucolytic and anti-oxidant properties. It is used for the treatment of pulmonary emphysema, bronchial asthma and COPD. The mode of action of fudosteine is similar to NAC. It donates/ releases the cysteine amino acid, which is essential for GSH production and increases overall endogenous cysteine. ${ }^{43}$ Fudosteine has higher bioavailability compared to NAC. Rhee et al examined the effect of fudosteine on mucin production. It was found that fudosteine down-regulated the expression of MUC5AC gene by inhibiting key signalling molecules (p-ERK in a bronchial cell line in vitro and p38 MAPK and ERK in the rat in vivo) ${ }^{44}$ and thus reduce mucus hypersecretion. ${ }^{44}$ Another study showed that fudosteine inhibited the peroxynitrite-induced oxidative stress by scavenging RNS, which is considered to be as another major contributor in the pathogenesis of COPD. ${ }^{45}$

\section{Procysteine}

Procysteine is a cysteine donating compound having higher bioavailability than NAC. Procysteine improves phagocytic function of macrophages by reducing glutathione-to-oxidized glutathione ratios (GSH/GSSG) in the lungs. Procysteine aids in reduction of IL- $1 \beta$ and TNF $\alpha$ production leading to improved macrophage function. ${ }^{46}$

\section{Nrf2 Activators}

Nrf2 is a transcription factor based on leucine zipper protein. It is associated with Keap 1 and is mainly found in the cytoplasm of the cell. It consists of a unique basic- leucine zipper (b-ZIP) domain that is important for DNA binding. It activates ARE-mediated Phase II detoxifying enzymes/genes and protects the body from oxidative and electrophilic stress. ${ }^{47}$ Therefore, Nrf2 is considered one of the several anti-oxidant targets that can attenuate oxidative burden in the lungs.

\section{Nrf2 Signalling}

The Nrf2 signalling pathway plays a pivotal role in protecting the cellular systems against oxidative burden or electrophilic stress by controlling the expression of various genes which are mainly engaged in detoxifying and eliminating the reactive free radicals and electrophilic agents. The lung is the main detoxification centre for ROS and hence Nrf2 expression is high in lungs. ${ }^{48} \mathrm{Nrf} 2$ activity is regulated by the cytosolic protein Keap1. In the absence of any oxidative stress, Nrf2 is maintained at a low level by its proteolytic degradation. In unperturbed cells, Nrf2 is associated with cytosolic protein Keap1 through its evolutionary conserved regulatory domain ${ }^{49}$. This association suppresses the activity of Nrf2 through the cul3-Rbx1 complex which ubiquitinates the Nrf2 and causes its constitutive proteasomal degradation. ${ }^{48}$ Keap1 contains highly reactive $\mathrm{SH}$ group, which is the main regulator of $\mathrm{Nrf2}$ stability. The protein DJ-1 is the master stabilizer of Nrf2 by inhibiting its association with Keap $1{ }^{50}$ When a cell encounters oxidative stress, Nrf2 dissociates from Keap1 followed by its stabilization and translocation into the nucleus. In the nucleus, it forms a heterodimer and interacts with several other transcription factors like Maf proteins. Subsequently, it associates with the cis element of ARE (antioxidant response element), that is present in the promoter region of the target gene. This enhances the expression of various phase II antioxidant and cytoprotective genes such as $\mathrm{NAD}(\mathrm{P}) \mathrm{H}$ quinoneoxidoreductase, glutathione S-transferase, UDP glucuronosyltransferase (UGT), heme oxygenase 1(HO-1), glutamate cysteine ligase, thioredoxin reductase 1 and glutathione reductase 9 (Figure 3)..$^{50}$

$\mathrm{Nrf} 2$ is a key player in protecting the body against various oxidative stress-related problems by switching on and off several genes and, hence it promotes cell survival by preventing apoptosis. ${ }^{51}$ Several studies has shown the disruption of Nrf2-Keap1 complex resulted in the upregulation of ARE controlled gene expression. Modification of the cysteine residue of Keap1 or phosphorylation of serine 40 of $\mathrm{Nrf} 2$ by oxidative stress-induced kinase resulted in Nrf2 nuclear translocation and its accumulation. ${ }^{51}$ One such study showed that ubiquitin-conjugating enzyme ubcM2 is helpful in maintaining the redox homeostasis by stabilizing the $\mathrm{Nrf} 2$ during the oxidative stress. $^{52}$ There are also some inhibitors reported, which suppressed the activity of Nrf2, such as Bach1 (Broad complex, Tramtarck, Bric-a-b-rac) protein that inhibited the Phase II detoxification by competing with Nrf2 for ARE binding sites and hence negatively controlling ARE associated gene expression. ${ }^{48}$

\section{Nrf2 Activating Drugs}

Nrf2 activation provides a very powerful approach to maintain the level of intracellular oxidants and neutralize the effect of free radicals induced by cigarette smoke in COPD. Various activators of Nrf2 play a pivotal role in reducing oxidative stress. Some of them are described below (Table 1). 


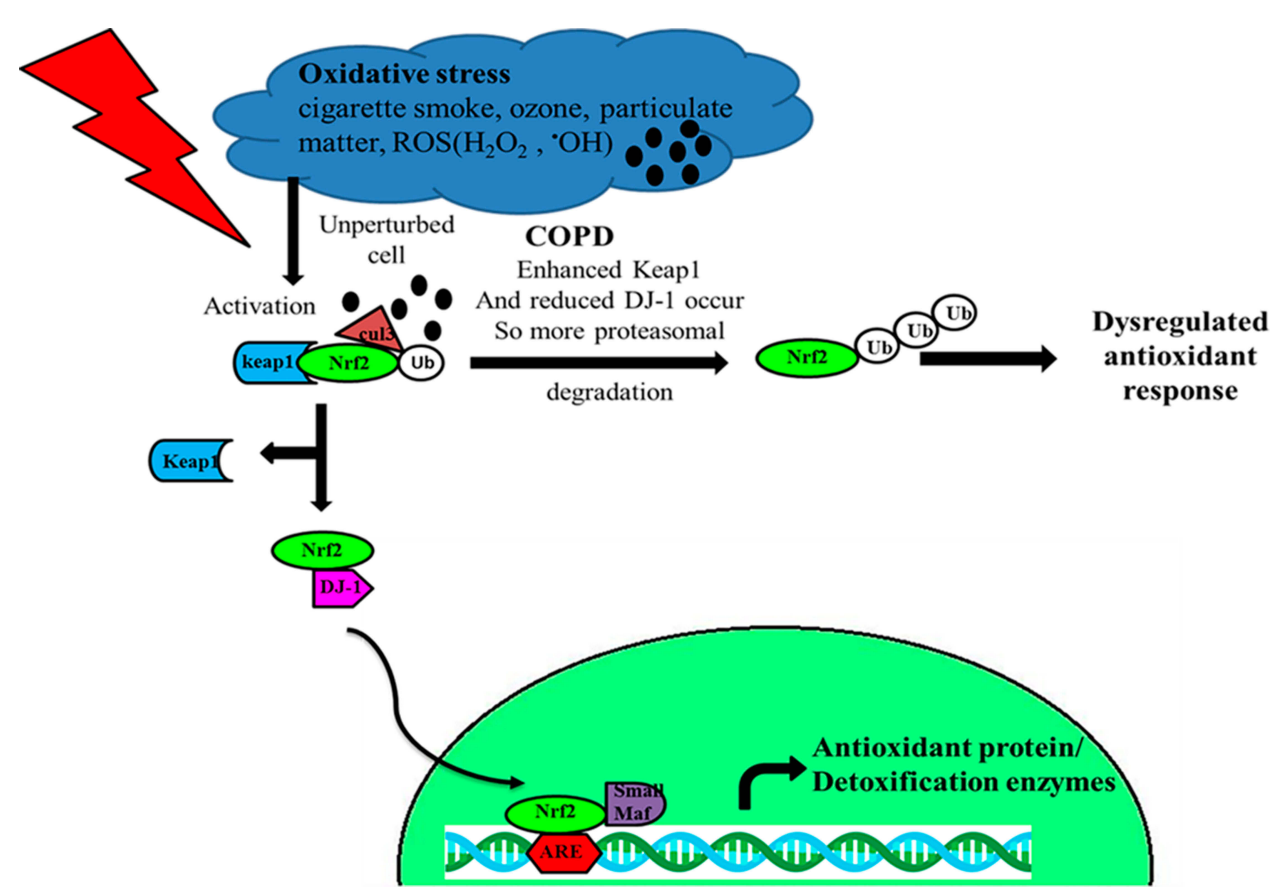

Figure $3 \mathrm{Nrf2}$ mechanism of action. Oxidative stress resulting from cigarette smoke, reactive oxygen species (ROS), particulate matter etc. leads to dissociation of Keap INrf2 followed by its stabilization and nuclear translocation, where it upregulates the various antioxidant genes that protect the body. In COPD, there is an increased Nrf2 proteasomal degradation as a result of increased Keapl production and reduction in master stabilization DJ-I protein.

\section{Sulforaphane}

Sulforaphane is an organosulphur compound mainly found in cruciferous vegetables such as broccoli sprouts, cauliflower, kale etc. It has the potential to detoxify and neutralize the free radicals, ROS produced and taken up by the body. Keum et al found that Nrf2 activated the ARE and enhanced the expression of HO-1 (an antioxidant enzyme).$^{53}$ In another study, it was demonstrated that sulforaphane induced the activation of Nrf2-dependent Phase 2 detoxifying enzymes and prevented the renal damage caused by oxidative stress resulting from ROS. $^{54}$

\section{Curcumin}

Curcumin is a yellow coloured compound that possesses both anti-oxidant and anti-inflammatory properties. It is found in turmeric. Rushworth et al found that curcumin enhanced the expression of protein kinase $\mathrm{C}$ (PKC) and p38 phosphorylation. It even assisted in the binding of Nrf2 transcription factor to ARE thereby increasing the availability and expression of cellular anti-oxidant enzymes such as HO- $1 .^{51}$ Curcumin administration of $100 \mathrm{mg} / \mathrm{kg}$ daily inhibited cigarette smoke-induced increase in inflammatory cells such as neutrophils and macrophages along with reduced emphysema/airspace enlargement. Hence, curcumin proved to be a potent inhibitor of elastase and cigarette smoke-induced pulmonary inflammation and emphysema in mice. ${ }^{55}$

\section{Resveratrol}

Resveratrol is a beneficial phytoalexin and a natural phenol found in the skin of red grapes, peanuts, and berries. It exhibit anti-carcinogenic, anti-inflammatory and anti-oxidant properties thus protecting the body against various stressors and damage. Resveratrol also protects $\mathrm{PC} 12$ cells from oxidative stress by inducing the activation of HO-1 via Nrf2-ARE signalling pathway. ${ }^{56}$ Kode et al in a study showed resveratrol treatment in A549 cells enhanced GSH and HO-1 levels through the Nrf2-ARE signalling. ${ }^{57}$

\section{Catechin}

Catechin is a flavonoid and a natural phenol that contains antioxidant properties. Studies conducted to examine the effect of epigallocatechin-3-gallate (EGCG) found enhanced nuclear translocation of Nrf2 and HO-1 gene expression preventing oxidative stress. ${ }^{58}$ In another study, they examined the effect of epicatechin obtained from cocoa and tea against brain ischemic damage that show enhanced expression of the neuroprotective HO-1 enzyme through the induction of $\mathrm{Nrf2} .{ }^{59}$

\section{Terpenoids}

Terpenoids are also known as isoprenoids (five carbon units). Various derivatives of terpenoids are used in reducing the 
Table I Activators of NRF2

\begin{tabular}{|c|c|c|c|}
\hline Antioxidant Compound & Structure & Mode of Action & References \\
\hline $\begin{array}{l}\text { Sulforaphane } \\
\text { (phytochemical) }\end{array}$ & & $\begin{array}{l}\text { - Suppressed the activation of p38MAPK } \\
\text { - Enhanced Nrf2 translocation to the nucleus and HO-I } \\
\text { expression. }\end{array}$ & {$[53,54]$} \\
\hline Curcumin & & $\begin{array}{l}\text { - Enhanced phosphorylation of PKC and p38 } \\
\text { - Enhanced activation of HO-I in monocytes }\end{array}$ & {$[55,64]$} \\
\hline Resveratrol & & $\begin{array}{l}\text { - Enhanced GSH production and inhibition of cytokine } \\
\text { release } \\
\text { - Enhanced Nrf2 translocation and HO-I gene } \\
\text { expression }\end{array}$ & {$[56,57]$} \\
\hline Catechin & & $\begin{array}{l}\text { - Enhanced nuclear translocation of Nrf2 } \\
\text { - Enhanced HO-I gene expression in B lymphocytes }\end{array}$ & [59] \\
\hline $\begin{array}{l}\text { Terpenoids (CDDO, Ginkgo } \\
\text { biloba) }\end{array}$ & & $\begin{array}{l}\text { - Decreased ROS levels } \\
\text { - Enhanced NrF2 nuclear translocation } \\
\text { - Enhanced HO-I gene expression }\end{array}$ & [62] \\
\hline Quercetin & & $\begin{array}{l}\text { - Enhanced GSH level and reduced ROS production } \\
\text { - Enhanced NrF2 nuclear translocation and HO-I gene } \\
\text { expression }\end{array}$ & [63] \\
\hline
\end{tabular}

oxidative stress-related damage through the activation of Nrf2 signalling. Sussan et al targeted Nrf2 with the triterpenoid CDDO imidazolide to significantly reduce the lung oxidative burden, alveolar destruction, and apoptosis caused by cigarette smoke. ${ }^{60}$ Another study examined the effect of dihydro-CDDO-trifluoromethyl amide on the cardiovascular damage via the inhibition of the oxidative burden. It was demonstrated that dihydro-CDDO-trifluoromethyl inhibited
ROS by activation of Nrf2. ${ }^{61}$ Extract from Ginkgo biloba also imparted protection in human endothelial cells treated with cigarette smoke extract-induced apoptosis. ${ }^{62}$

\section{Quercetin}

Quercetin is a plant pigment (flavanoid) found in many fruits and vegetables such as red wine, apple, onion, berries, Ginkgo biloba etc. It activates Nrf2 mediated HO-1 
gene expression and protects human hepatocytes from oxidative burden as shown by Yao et al ${ }^{63}$ However, further clinical and experimental studies that supports the beneficial role of quercetin are needed to establish their therapeutic potential in COPD.

\section{Enzyme Antioxidant}

Reactive oxygen species produced inside the cells can be effectively removed by the enzymatic anti-oxidants such as catalase, superoxide dismutase (SOD), glutathione peroxidase (GPx) whose expression and functions are altered during oxidative stress. Hence, to maintain the balance between oxidant-anti-oxidant systems, enzyme mimic can be used. Enzyme mimic imitates the normal function of the enzyme by possessing catalytic activity.

\section{SOD (Superoxide Dismutase) Mimetics}

Three classes of enzyme mimetics are reported. Several manganese-based macrocyclic ligands such as M40401 and M40414 belong to the first class of SOD enzyme mimetics. ${ }^{65,66}$ The second class comprises of manganese metalloporphyrins, which consist of AEOL-10113 and AEOL-10150. ${ }^{67,68}$ The third class comprises of 'Salens'. Salens possess both superoxide dismutase and catalase activity. Thus, it can neutralize a broad range of free radicals such as superoxide anion $\left(\mathrm{O}_{2}{ }^{-}\right)$, hydrogen peroxide $\left(\mathrm{H}_{2} \mathrm{O}_{2}\right)$ and peroxynitrite $\left(\mathrm{ONOO}^{-}\right)$radicals. ${ }^{69}$ It has been found that M40419 significantly reduced the oxidative stress markers expressed in the lung of animal models. ${ }^{65}$ AEOL-10150 has also been observed to inhibit cigarette smoke-induced inflammatory response, decreased the production of $\mathrm{ONOO}^{-}$and lipid peroxidation. ${ }^{68}$

SOD3 is an extracellular superoxide mostly found in the lungs that is exposed to free radicals as compared to most other tissues. ${ }^{70}$ SOD3 has the ability to capture superoxide anion $\left(\mathrm{O}_{2}\right)$, therefore it plays a crucial role in protecting the lung against damage caused by oxidative stress. Previously, SOD3 has been reported to protect the mouse lung from cigarette smoke and elastase-induced pulmonary emphysema by attenuating the fragmentation of ECM and modification of elastin fragments caused by oxidative stress. ${ }^{71}$ Another study showed that SOD3 can effectively decrease the oxidative burden in mouse macrophages. ${ }^{72}$ These results showed that the development of enzyme mimetics is a powerful therapeutic approach to treat COPD and emphysema.

\section{Glutathione Peroxidase Mimetics}

Ebselen compounds have a neutralizing effect against $\mathrm{ONOO}^{-}$radical and thus act as strong anti-oxidants. They are selenium-based compounds mimicking glutathione peroxidase activity ${ }^{73}$. Ebselen reduces the LPS-induced inflammation by inhibiting the TNF- $\alpha$ and IL-1 $\beta$, which are mainly involved in the recruitment of inflammatory cells such as neutrophils. It also inhibits the expression of ICAM-1 ${ }^{74,75}$ These studies show that the enzyme mimetic is beneficial by inhibiting lung inflammation that is associated with the oxidative burden.

\section{Spin Traps}

Spin traps are agents that have the ability to capture ROS and convert them to a stable form. Most of the spin traps are made of nitroxide nucleus. They have been widely used in both in vitro and in vivo studies. The compound BN 80933 (a-phenyl-N-tert-butyl) reacts with the reactive radicals at the site of inflammation and convert them into the stable end forms. ${ }^{76}$

\section{Importance of Nanotechnology in COPD}

Submicron size particles with unique innate physical properties such as small size, large surface area to volume ratio, biocompatibility and biodegradability are defined as nanoparticles. ${ }^{77}$ These properties allows them to have better contact with the neighbouring tissues and cells thereby increasing the efficiency of cellular drug and gene delivery. ${ }^{78}$ There are several benefits when we use nanoparticles as drug delivery systems against chronic inflammatory lung diseases. They provide sustained release of the drugs and help in overcoming airway defenses while simultaneously targeting the diseased cells. For example, Janib et al demonstrated that STEALTH liposome encapsulating budesonide, a potent corticosteroid, controlled the release of the drug and enabled the therapeutic concentrations to be retained for longer periods in rat lungs while reducing the systemic toxicity. ${ }^{79}$ Combination treatment of drugs can also be delivered using nanoparticles. Van Rijt et al developed liposomes comprising of two drugs, beclomethasone (glucocorticoid steroid) and formoterol (beta 2 -selective receptor agonist). Beclomethasone retained its effect for longer times and formoterol enhanced lung function and augmented peripheral lung deposition without affecting mucociliary escalator. ${ }^{80}$ Therefore, the development of advanced cargo carrying nanocarriers would be vital to treat chronic lung disease such as COPD/emphysema. 


\section{The Rationale Behind the Development of Multifunctional Nanocarriers for COPD}

Size

Studies show that both charge and size plays an essential role in the mucociliary clearance of particles. The major challenge in using nanocarriers for treatment of chronic obstructive lung disease is their penetration across the mucus layer since mucus layer in the COPD is not only too thicker, but is highly viscoelastic. ${ }^{81}$ Rytting et al demonstrated that small polystyrene nanoparticles with an average diameter of $120 \mathrm{~nm}$ move readily across the sputum of the cystic fibrosis patients. On the contrary, larger particles with a diameter of $270-250 \mathrm{~nm}$ had comparatively fewer mobility. ${ }^{82,83}$ In addition surface modifications of the nanoparticles such as PEGylation, which renders the nanoparticles with neutral charge to significantly enhance the delivery of therapeutics across the mucosal barrier ${ }^{84}$ and have an improved bronchial clearance of the particles. ${ }^{85}$ The type of disease influences the particle uptake and its localization. For an instance, Geiser et al showed that inhaled gold nanoparticles were taken up by surface macrophages of alveolar type 1 epithelial cells in Scnn1b-transgenic mice with COPD/emphesema at a higher rate compared to that of wild type mice. ${ }^{85}$ Particles with size less than $10 \mathrm{~nm}$ are likely to be eliminated by the reticuloendothelial system (RES) via glomerular capillary by liver, spleen and kidney and if the particle size is more than $100 \mathrm{~nm}$, they are likely to be cleared by the alveolar macrophages. Thus, particle size of nanocarriers is crucial in developing and determining the effectiveness of the system. ${ }^{86,87,88}$

\section{Particle Shape}

Distinct geometries of the particle influence the cellular uptake, hemorheological diameter, and in vivo cell fate. More than spherical particles the discoidal particles display unique plummeting and migration dynamics that often favour vessel wall interaction. ${ }^{87,89}$ Shape also affects circulation half-life of the particle, such as, filamentous polymer micelles are known as filomicelles possess a long circulation lifetimes ( $>1$ week after administration) than spherical particles that had a lifetime of only 2-3 days, giving these particles a larger tendency to align with the blood flow as shown by Champion et al. ${ }^{90}$ Uptake of the nanoparticles was also affected by geometrical parameters such as the aspect ratio and curvature of the nanoparticles with regard to the macrophage internalization. Champion et al demonstrated the particle shape at the point of contact from nanoparticles to the cells. ${ }^{91}$ These findings demonstrated the effect of curvature on the kinetics of the phagocytosis with the particles that possessed, normalized curvature length $(\Omega) \leq 45^{\circ}$ underwent faster internalization than particles with $(\Omega) \geq 45^{\circ}$. These results demonstrated that ellipsoidal, cylindrical and discoid particles and all constructs possessing high aspect ratio and minimal regions of curvature have enhanced accumulation of therapeutics. $^{92}$

\section{Surface Charge}

Surface charge is another important attribute of the nanoparticles to be used as nanocarriers. It can be modified for improving the circulation lifetime and affects the accumulation of the nanocarrier at the specific site of interest. It has been demonstrated that nanoparticles with neutral and negative surfaces often have longer circulation half-lives due to a reduction in adsorption of serum proteins. On the other hand, positively charged nanoparticles were shown to be uptaken non-specifically by the majority of the cells. However, positively charged nanoparticles can facilitate endosomal release through a mechanism called proton sponge effect that hinders the degradative effects of the endosomal pathway compartments on the drug freight as illustrated by Nel et al. ${ }^{93}$ Therefore, for the successful delivery of nanoparticles to the target site, a negative surface charge is desired along with a switch to make it positive on the arrival at the target site.

\section{The Rationale Behind the Design of Nanocarriers for Drug Delivery to the Lungs}

The advantages of using nanocarriers for delivery of drugs include enhanced drug accumulation at the target site, prolonged drug circulation time, minimal drug degradation as well as the ease of drug administration at the site of interest. ${ }^{94}$ Nanocarriers facilitate controlled release of the drug at the target site. When coupled with ligands against specific receptors expressed on the diseased cells such as folate receptors in case of breast cancer cells, nanocarriers enable specific cell targeting. Therapeutic molecules such as drugs, siRNA (small interfering RNA), DNA and peptides can be encapsulated inside nanocarriers using various techniques depending on its physical and chemical properties. It can further be retained by covalent and electrostatic interactions between the surface of the nanocarrier and 
therapeutic molecules. Not all drugs are compatible with nanoparticles, which emphasize the need for surface modifications of nanoparticles. Nanoparticles have distinct properties as well as drug release kinetics depending on the materials used for the fabrication and modifications made on their surface. ${ }^{95,96}$

Nanocarriers interact with the biomolecules present on the cell surface as well as within the cell. For instance, due to excellent biocompatibility and the possibility of surface modifications, biodegradable polymeric nanoparticles have been used as an effective drug carrier for the sustained release of the encapsulated drug. They have been studied substantially for their ability to deliver drugs that include various pulmonary formulations for COPD, asthma, cancer and tuberculosis therapy. ${ }^{97-99}$ Various liposomal formulations have been explored for the delivery of drugs to the lungs. There are several liposomal drug delivery systems available in the market approved by FDA and several liposomal-based formulations are in the clinical trials .100,101 Some properties to be considered while designing nanocarriers for drug delivery into the lungs are discussed below.

\section{Surface Modifications}

Surface modification of the particles has an effect on their bio-distribution and circulation times. Hydrophobicity of the nanocarrier determines the extent to which a particle can bind to the blood components. Particles that are nonsurface modified and hydrophobic are cleared rapidly. ${ }^{102}$ Circulation time of the particles in the blood can be enhanced by coating the particles with hydrophilic polymers or surfactants such as polyethylene glycol (PEG). These decorated nanoparticles reduce phagocytosis. ${ }^{103}$ Moreover, PEGylation increases mucous penetration for chronic obstructive lung diseases. Modification with chitosan often creates more mucoadhesive nanoparticles thus providing prolonged circulation times. ${ }^{104}$ This increases the drug uptake and bioavailability which is beneficial in treating obstructive lung diseases such as lung cancer and allergic asthma. Moreover, in addition to the chemical modifications, there can be surface modifications by biological fluids such as by the formation of protein corona that can change the particle properties. ${ }^{105}$ Inhalable nanoparticles are often coated by the lung surfactant phospholipids, thus changing their toxicity and cellular uptake. ${ }^{106}$ The mode of binding and binding strength of lung surfactants to the nanoparticles mainly depends on the particle surface. $^{107}$

\section{Targeting}

There are various possibilities offered by nanoparticles for specific in vitro and in vivo targeting. Generally, two ways are there which can direct the nanoparticles towards the diseased site (For example, active targeting and passive targeting). Passive targeting occurs by enhanced permeation and retention effect (EPR) (Figure 4). This involves the accumulation of nanoparticles in tissues due to the formation of unstructured and leaky blood vessels resulting from inadequate ejection and poorly regulated angiogenesis, especially in tumours. It can also be achieved by the presence of the surface charges on nanoparticles. For instance, Hashizume et al demonstrated that positively charged paclitaxel loaded liposomes targeting the endothelial cells in solid tumours in patients suffering from advanced cancer and liver metastasis. ${ }^{108}$

Active targeting involves attachment of ligands such as peptides and antibodies to the surface of the nanocarriers specific to the receptors present on the diseased cells on the

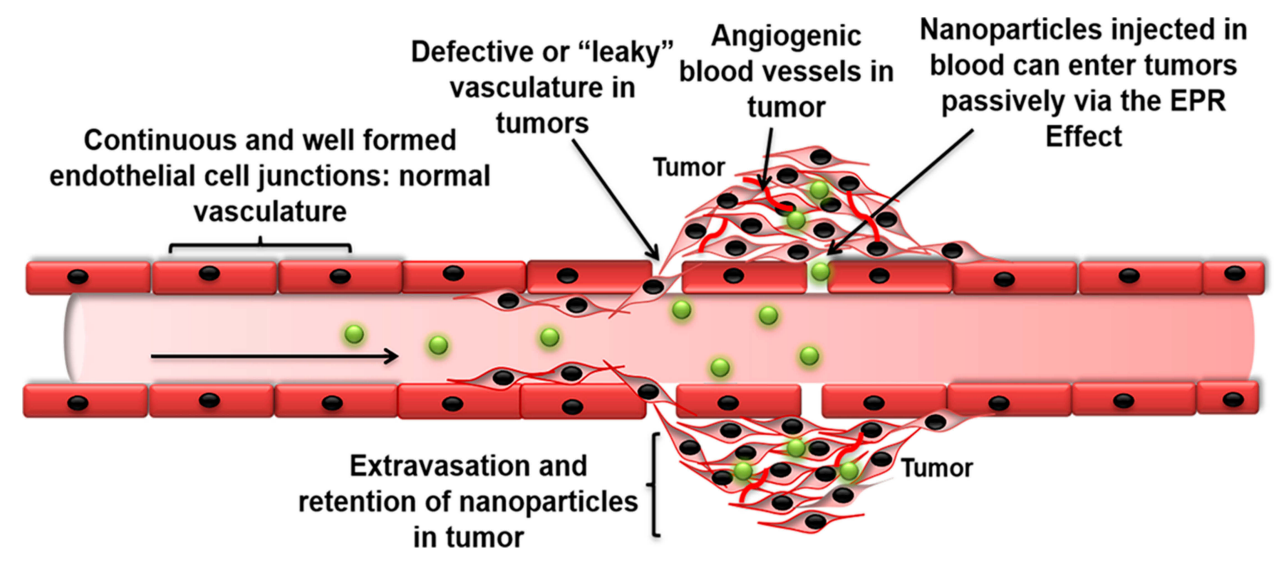

Figure 4 Schematic representation of the EPR Effect. 
surface of the nanocarriers. For example, integrins are one such transmembrane receptors that mediate interactions of the cell with the surrounding extracellular matrix and are upregulated in a proliferating tumour cell. ${ }^{109}$ Liposomes targeting integrins that are loaded with doxorubicin suppressed the tumour growth especially in a mouse model of colon carcinoma whereas nonspecific targeting of liposomes did not yield significant results. Another example of a receptor that can be used in targeted delivery is folate receptor, which is overexpressed in a variety of tumours especially breast cancer cells as demonstrated by Yoo et al. ${ }^{110}$ Moreover, doxorubicin-loaded folate receptor-targeted polymeric micelle revealed increased cellular uptake along with enhanced circulation times. Other examples of cell receptors that are used for particle delivery includes epidermal growth factor receptor (EGFR) and transferrin receptor. ${ }^{111}$ Cell adhesion antibodies can also be conjugated to achieve specific cell targeting. ${ }^{112}$

\section{Controlled Drug Release}

Controlled release of the drug from the nanocarrier is a crucial prerequisite for therapeutic success. Sustained release or stimuli-responsive release can enhance the efficiency of the drug. Most of the polymeric nanoparticles and liposomes have exhibited sustained and continuous release of the drug either by diffusion of the drug outside the particle or by slow degradation of the particle over time, triggering the release of drugs. Stimuli-responsive controlled drug release can be accomplished either by a change in biological environments such as a change in $\mathrm{pH}$ (e.g. endocytic pathway compartments) or reducing environment of the cell or altered conditions mediated by the disease-specific enzymes in the cells. ${ }^{113}$ For example, polymeric nanoparticles that contain $\mathrm{pH}$ sensitive linkers can change their conformation in response to $\mathrm{pH}$ changes of the cellular environment, which then triggers the release of drugs. The redox potential gradient between the oxidizing extracellular and reducing intracellular environment of the cancerous cells have also been explored for the biodegradation of the polymer. Another example for the $\mathrm{pH}$-responsive drug release, is the PEGPeptide-Lipid conjugates, where PEG molecules could be cleaved from the carriers by matrix metalloproteinases (MMPs) that are overexpressed in tumours. ${ }^{114}$ Controlled release of the therapeutic cargo can also be achieved by external physical stimuli such as heat, light, ultrasound magnetic or electric field. An example of this includes ultrasound microbubbles, which trigger modulated drug release in response to ultrasonic waves and aerosol droplets consisting of super-paramagnetic iron oxide nanoparticles which are directed using magnetic field as a physical stimuli. ${ }^{115,116}$

\section{Various Nanoparticles for Chronic Lung Diseases}

Diverse materials can be used for the fabrication of nanocarriers for chronic lung diseases such as organic and inorganic materials comprising proteins, synthetic/natural polymers, lipds, and metals. These nanoparticles can be classified into various groups on the basis their components and characteristic dimensionalities such as liposomes, micelles, polymeric nanoparticles, dendrimers, solid-lipid nanoparticles, proteinbased nanoparticles and inorganic nanoparticles (Figure 5). ${ }^{117}$

\section{Liposomes}

Most widely used lipid-based drug carriers are liposomes. They are spherical vesicles consisting of single lipid bilayer membrane or several lipid bilayer membranes. The outer surface of the liposome is generally modified by polymers such as polyethylene glycol (PEG). Such coating extends the blood circulation time of the liposomes by reducing the uptake by the phagocytic system. Such liposomes are popularly known as STEALTH liposomes. This STEALTH technology has enabled a large number of effective liposomal formulations consisting of active molecules with high targeting efficiency and activity. Generally, liposomes within the range of 50-500 $\mathrm{nm}$ are suitable for drug delivery. Neutral, anionic and cationic liposomes can be fabricated by varying composition of the liposomal membrane. ${ }^{118}$ Figure 6 describes a multifunctional liposomal hybrid carrier. ${ }^{119}$

Liposomes have extensively been used as the agents for aerosol delivery of drugs, nucleic acids (DNA/RNA) and vitamins targeting various diseases ${ }^{120}$. There are several techniques that are used for the nebulization of liposomes as dry powder inhalers such as freeze drying, spray drying, spray freeze drying help in achieving long-term stability and overcoming the problems associated with suspension drugs.121 Liposomes have several advantages to be used as dry powder inhalers for lungs such as they retain their size, drug payload and do not aggregate after aerosolization. Moreover, they even show enhanced accumulation in the lungs, appropriate retention for a longer period of time and penetrate into the lung cells after inhalation which is followed by the controlled release of the active payload such as drug cargo inside the cells. ${ }^{122}$ Negatively charged and neutral liposomes have known to have enhanced biocompatibility as compared to the cationic liposomes. They are normally used in conjugation 


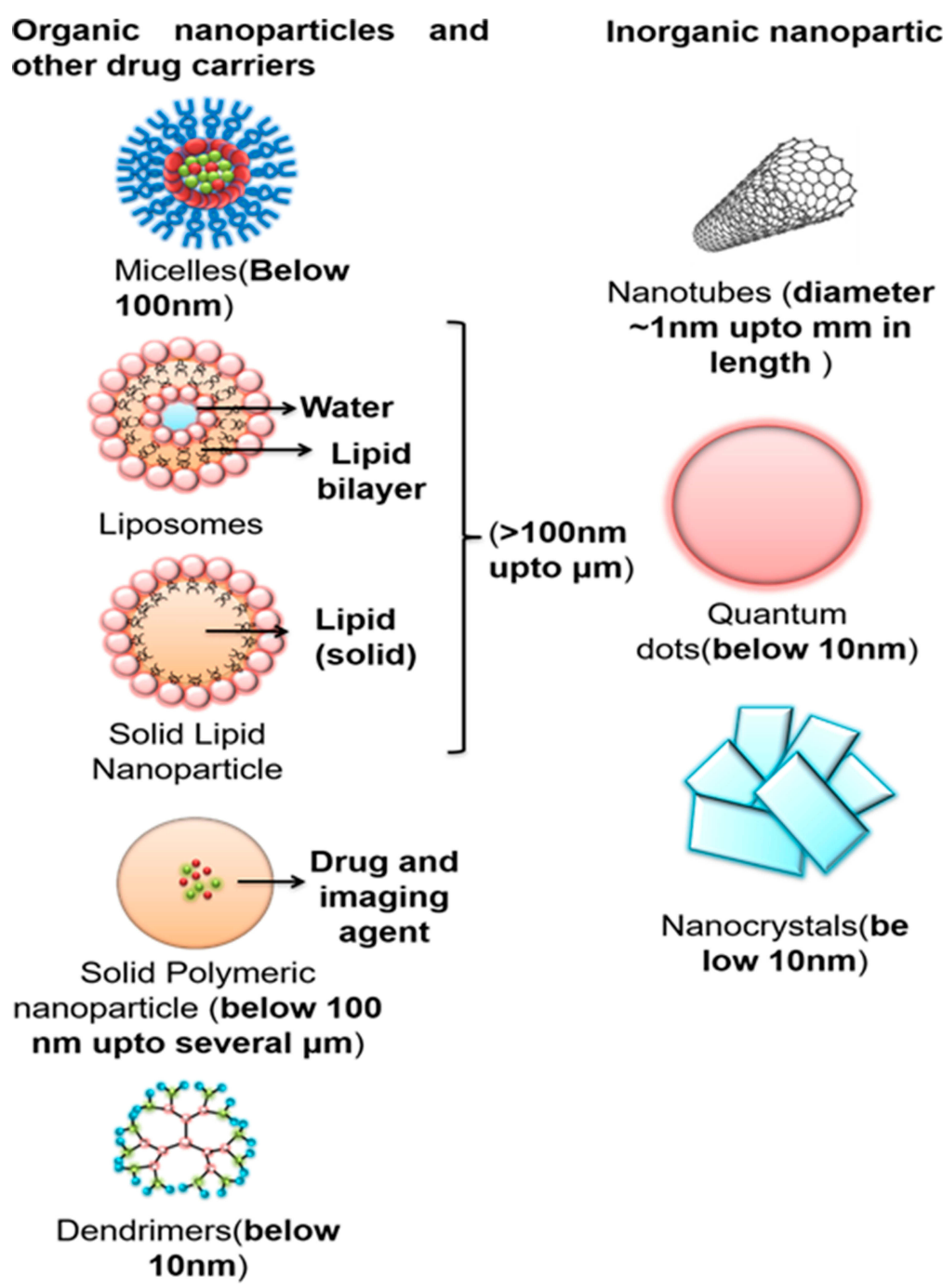

Hybrid nanoparticles

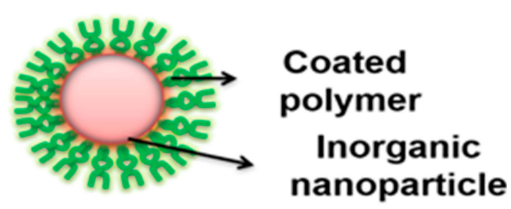

Polymer coated inorganic nanoparticles(below10 $\mathrm{nm}$ )

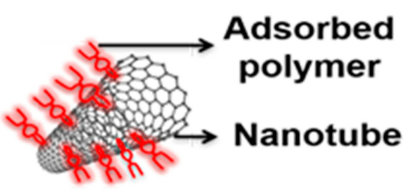

Adosrbed polymer on nanotubes (below 10nm)

Figure 5 Various nanocarriers for drug delivery to the lungs.

with the negatively charged nucleic acids, as such kind of modifications usually prevents the adverse effects on the cells. ${ }^{121,123}$ It has been found that negatively charged anionic liposomes can be easily internalized by the macrophages and is proven to be an effective approach to target alveolar macrophages in COPD patients because these cells play a key role in COPD pathogenesis. A liposomal dexamethasone delivery system coupled with an antibody targeting surfactant protein A was developed by Li et al. ${ }^{124}$ This antibody is abundantly expressed by the type II alveolar epithelial cells in rats with acute respiratory distress syndrome (ARDS). It was demonstrated that compared to the rats receiving free dexamethasone sodium phosphate, these liposomal steroids had a better therapeutic effect and fewer side effects. Li et al prepared ciprofloxacin loaded liposomes with a high encapsulation efficiency of ciprofloxacin using gradient ammonium sulfate method. ${ }^{124}$ These liposomes were efficient in delivering the ciprofloxacin to the target site while minimizing the irritation effects. Further, Cipolla et al also created liposomal formulations in which they encapsulated ciprofloxacin nanocrystals. These formulations were freezedried, thawed and subsequently aerosolized. ${ }^{125}$ It was observed that these formulations after nebulization were stable for a longer period of time.

\section{Solid Lipid Nanoparticles and Lipid Hybrid Polymer Nanoparticles}

Lipid-based nanoparticles can be easily fabricated from the lipid-like materials such as cholesterol and phosphatidylcholine as they are biocompatible. These nanocarriers 


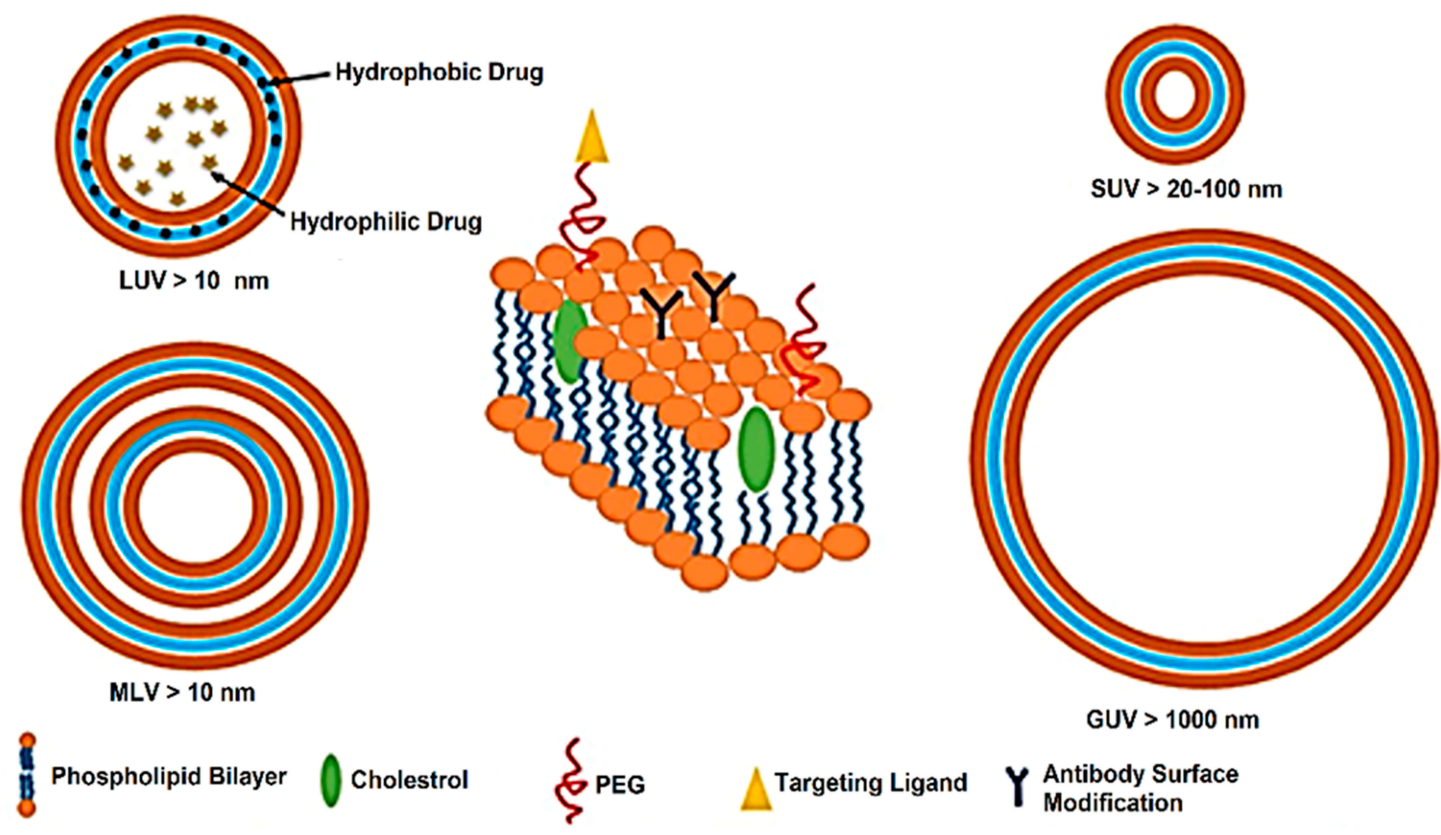

Different Lipososme types based on size and Lamellarity. Also shown is a portion of a typical lipid biliayer with multifunctional surface modification

Figure 6 Liposomal nanocarriers, Copyright 2015, American Chemical Society. ${ }^{\prime \prime \prime}$

are able to carry large amounts of drugs and the outer lipid layer present in these carriers contribute to easy cellular uptake. Examples of such lipid-based nanocarriers are Dipalmitoylphosphatidylcholine (DPPC) and Dipalmitoylphosphatidyl ethanolamine-methoxy(polyethene glycol) (DPPE-PEG) which are often used for drug delivery to the lungs. Meenach et al developed a micro/nanoparticle system comprising of DPPC/DPPE-PEG dipalmitoylphosphatidylcholine (DPPC) and dipalmitoylphosphatidylethanolamine PEG (DPPE-PEG) in the form of dry powder inhalation aerosol for the delivery of paclitaxel to the lungs which is an anti-cancer drug. ${ }^{126}$

Polymers can also be used to develop lipid-polymer hybrid nanoparticles apart from modification of the lipid nanoparticles. This approach is successfully used as an alternative to other lipid-based nanoparticles comprising even liposomes. For example, Wang et al successfully developed hybrid Poly (lactic-co-glycolic acid) (PLGA) nanoparticles that were enveloped by phosphatidylcholine (PC) and were used for drug delivery to the lungs. ${ }^{127}$ These nanoparticles had a spherical morphology and were adsorbed onto the chitosan carrier. Moreover, the aerodynamic diameter of these particles should be between $1-5 \mu \mathrm{m}$ since nanoparticles with a diameter smaller than $1 \mu \mathrm{m}$ could be exhaled back while the particles larger than $5 \mu \mathrm{m}$ could be accumulated in the mouth instead of lungs. Porous nanoparticles have a lower aerodynamic diameter than non-porous nanoparticles as demonstrated by Sinha et al who used Poly-lactide-coglycolide porous nanoparticles for the delivery of voriconazole to the lung cells. ${ }^{128}$ Recently, nanoparticles loaded with anti-cancer drugs, antibiotics, anti-asthmatic drugs and antioxidant agents, have been used successfully for treating chronic inflammatory lung diseases.

Lipid nanoparticles are a new generation of nanoparticles also called as nanostructured lipid carriers (NLC). They are appropriate for inhalation delivery of different pulmonary drugs and nucleic acids such as siRNA. ${ }^{129}$ The inner core of the NLC can be successfully loaded with the lipophilic drugs. Moreover, these particles can be made positively charged by coating them with a cationic lipid(s). Negatively charged nucleic acids can easily form complexes with these cationic nanoparticles. On the surface of NLC thiol-modified DNA or RNA molecules can be conjugated by biodegradable disulphide (S-S) bonds. In addition, PEG polymer is used to modify the surface of NLC and they can be coupled with the targeting moieties. Pitard et al demonstrated that exceptionally high therapeutic efficiency of NLC was achieved when loaded with paclitaxel that caused cell death induction. It was also observed that siRNA targeted at MRP1 and BCL2 genes 
suppressed cancer cell resistance and anti-apoptotic defense that was used for inhalation chemotherapeutic treatment of lung cancer. ${ }^{130}$

\section{Polymers}

Polymers are the macromolecules that are formed by repeating units called monomers. Polymers are a major part of the therapeutics that can be used to deliver drugs via inhalation to the lungs. A variety of polymeric drug delivery systems can be created by different types of polymers that consists of linear polymers coupled with the active components such as drugs, nucleic acids, targeting moieties etc. either directly or via spacers of different designs. Limited numbers of polymers are available for the local pulmonary inhalation delivery. One such example of aerosol dry powder formulation is Poly(lactic-co-glycolic) and poly(ethylene glycol)-co-poly(sebacic acid) which are suitable for inhalation delivery. ${ }^{131}$ There are two types of polymers used for the synthesis of polymeric nanocarriers. They are natural and synthetic polymers:

Natural polymers consisting of polysaccharides and proteins generally obtained from natural and living sources. They are biodegradable, biocompatible, nontoxic and non-immunogenic in nature. Starch, alginate, chitosan, hyaluronic acid and dextran are examples of polysaccharides whereas albumin and gelatin are the widely used proteins. Synthetic polymers such as poly (lactide-co-glycolide) (PLGA), polyacrylates and polyanhydrides are easy to produce and least likely to be contaminated. Depending on the chemical nature, each polymer has a unique property. For example, PEG is a polymer that is bioinert in nature. PEGylation makes nanoparticles biocompatible and reduces opsonisation of nanoparticles by immune cells so that they are able to penetrate the respiratory mucus. Cationic polymers due to electrostatic affinity such as polyethyleneimine (PEI) bind to nucleotides. PEI-based nanoparticles have been effectively used for gene delivery to treat chronic lung diseases. ${ }^{132}$ A variety of nanocarriers are developed and have been successfully experimented for the treatment of chronic lung diseases including asthma, COPD, pulmonary hypertension and tuberculosis. Vij et al developed a multifunctional polymeric vesicle for the combined delivery of COPD drugs especially the corticosteroid prednisolone and/or the anti-inflammatory bronchodilator theophylline for the treatment of chronic lung diseases. This polymeric vesicle is formed by mixture of PLGA and poly(ethylene glycol). ${ }^{133}$

\section{Dendrimers}

Dendrimers are the molecules having a repeated branched structure. The size of dendrimers varies from 4 to $20 \mathrm{~nm}$ and they are mostly used for drug delivery. Dendrimers briskly penetrate the circulation system curtailing their retention in the lungs. For instance, Kaminskis et al showed anti-cancer efficacy of the doxorubicin conjugated $56 \mathrm{kDa}$ PEGlyated poly-lysine dendrimers and these dendrimers are rapidly removed from the lungs after the intra-tracheal instillation. ${ }^{134}$ Around $15 \%$ of the administered drug was retained in the lungs. Dendrimers are a part of the more complex delivery systems that can potentially be used for inhalation delivery. For example, dendrimers containing the surface charges were successfully employed for the delivery of nucleic acids. Patil et al successfully designed the triblock poly(amido amine)-poly(ethylene glycol)-poly-1lysine (PAMAM-PEG-PLL) nanocarriers consisting of a whole cationic charge and enabled the cytoplasmic delivery of siRNA. ${ }^{135}$ Khan et al demonstrated the potential of dendrimer nanomaterials for the delivery of siRNA using a combinatorial approach where free amine on multigenerational poly(amido amine) and poly(propylenimine) dendrimers were replaced with alkyl chains of increasing length. These were designed to target Tie2-expressing lung endothelial cells in vivo. It was observed that even at high doses, these dendrimers-lipid derivatives were non-toxic as they did not cause a significant increase in proinflammatory cytokines. ${ }^{136}$

Generally, dendrimers are highly mono dispersed nanoparticles whose size and the functionality of the formulation are controllable. They represent an effective system that can be used for delivery of a variety of drugs, antibiotics, and steroids and anti-cancer agents. Bohr et al, in a recent study, demonstrated that the phosphorous dendriplexes could effectively deliver siRNA to lungs. The phosphorous-based dendrimers with either pyrrolidinium or morpholinium as terminal protonated amino groups were chosen. It was demonstrated that pyrrolidinium dendriplexes due to the high Pka value had a higher siRNA complexation. It improved protection against enzymatic degradation that resulted in a higher cellular uptake as well as a high in vitro silencing efficiency of TNF $\alpha$ in the LPS in-activated mouse macrophage line RAW264.7, compared to morpholinium-containing dendriplexes (Figure 7). ${ }^{137}$ 

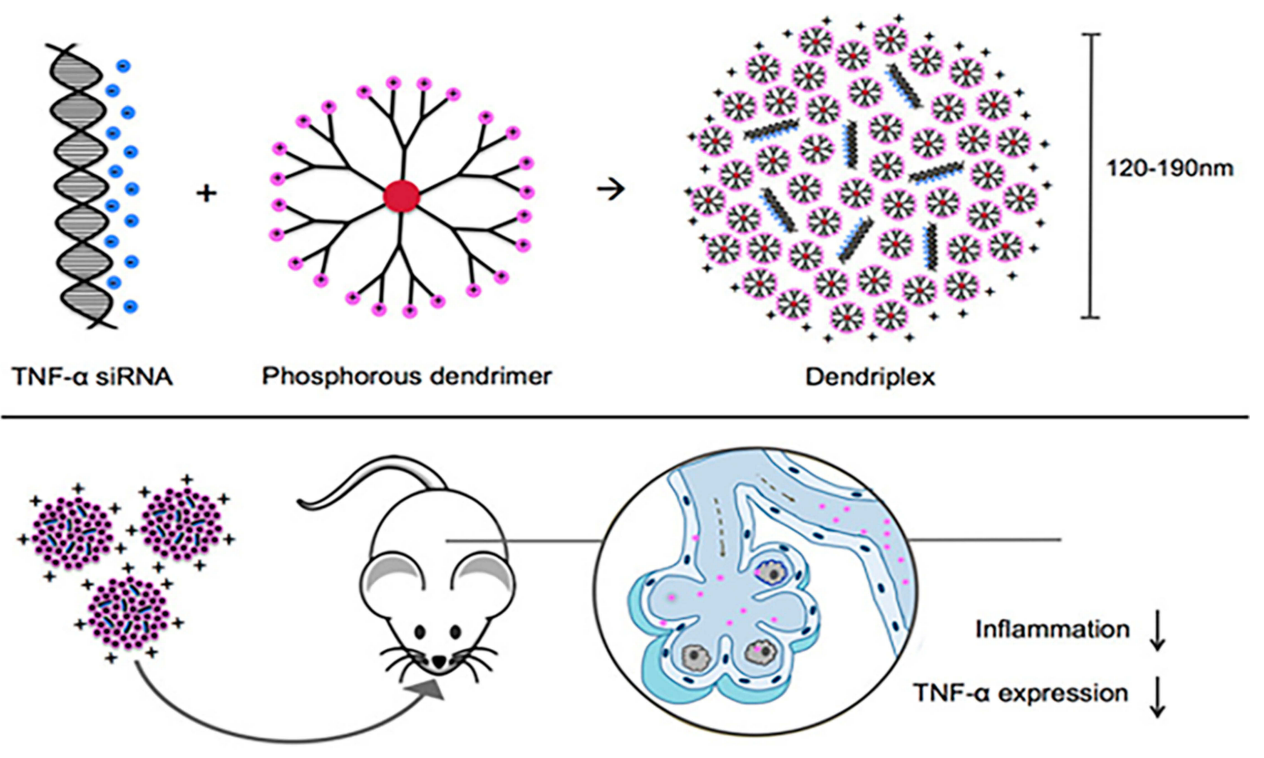

Intranasal delivery to inflammed lungs

Figure 7 Pyrrolidiniumdendriplexes Copyright 2017, American Chemical Society. ${ }^{128}$

\section{Nanospheres}

Nanospheres are nanocarriers of varying size from 10-200 $\mathrm{nm}$, mainly designed for the purpose of delivering gene and drugs. They are amorphous or crystalline in nature and have the ability to protect the drugs from enzymatic and chemical degradation. Several biodegradable nanospheres include albumin nanospheres, gelatin nanospheres, dextran nanospheres, and polylactic acid nanospheres. Pitard et al successfully synthesised a nanosphere using four poly-ethylene oxide/polypropylene oxide blocks centred on an ethylenediamine moiety consisting of positive charges which were easily coupled with nucleic acids/plasmids. This nanosphere successfully delivered chemokine fractalkine as the chemotherapeutic agent. ${ }^{130}$ Furthermore, in vivo testing by using experimental lung metastasis model also supported nanospheres use as a favourable immunotherapeutic approach. Jiang et al also developed paclitaxel loaded chitosan hollow nanospheres that were fabricated using polystyrene nanospheres as templates. These nanospheres offered a sustained released of paclitaxel as well as good bioavailability. It inhibited the proliferation of A549 cells and enhanced apoptosis. ${ }^{138}$ Further, Price et al also developed nano-in microparticles (NIMs) containing superparamagnetic iron oxide nanoparticles (SPIONs) with either doxorubicin or fluorescent nanospheres as dry powder inhalers. In vivo targeting of inhaled NIMs was also assessed in the presence of an external magnetic field using a healthy mouse model $^{139}$ (Figure 8).

\section{Inorganic Nanoparticles}

Inorganic substances such as metal and metal oxide nanoparticles (gold, silver, silica cerium oxide and iron oxide) are widely used for development of nanotherapeutics. Because of the unique plastic and magnetic properties, inorganic nanoparticles such as gold nanoparticles (AuNPs) and iron oxide nanoparticles are generally used as imaging contrast agents in a variety of techniques such as computed tomography (CT), magnetic resonance (MR) or positron emission tomography (PET). Inorganic nanoparticles are also commonly being explored as a platform for diagnostics and therapeutics. Gold nanoparticles have extensively been explored for the purpose of gene delivery as gold is a cationic metal ion and it easily binds to the negatively charged DNA and RNA molecules. Geiser et al synthesised inhalable gold nanoparticles that bind to the alveolar epithelial cells in a COPD mouse model. ${ }^{85}$ Despite all the advantages, higher levels of toxicity that is posed to cells by the use of metallic inorganic nanoparticles remain a cause of major concern. Gold nanoparticles form aggregates when they are intravenously injected beside the fact that they bind to positively charged serum proteins. However, a recent study has revealed that surface modifications by PEG can prevent aggregation in gold nanoparticles. However, gold nanoparticles have low excretion that hinders their clinical applications and long-term studies. Recently, Fytianos et al developed a fluorescently encoded poly(vinyl alcohol) (PVA)-coated gold nanoparticles, 


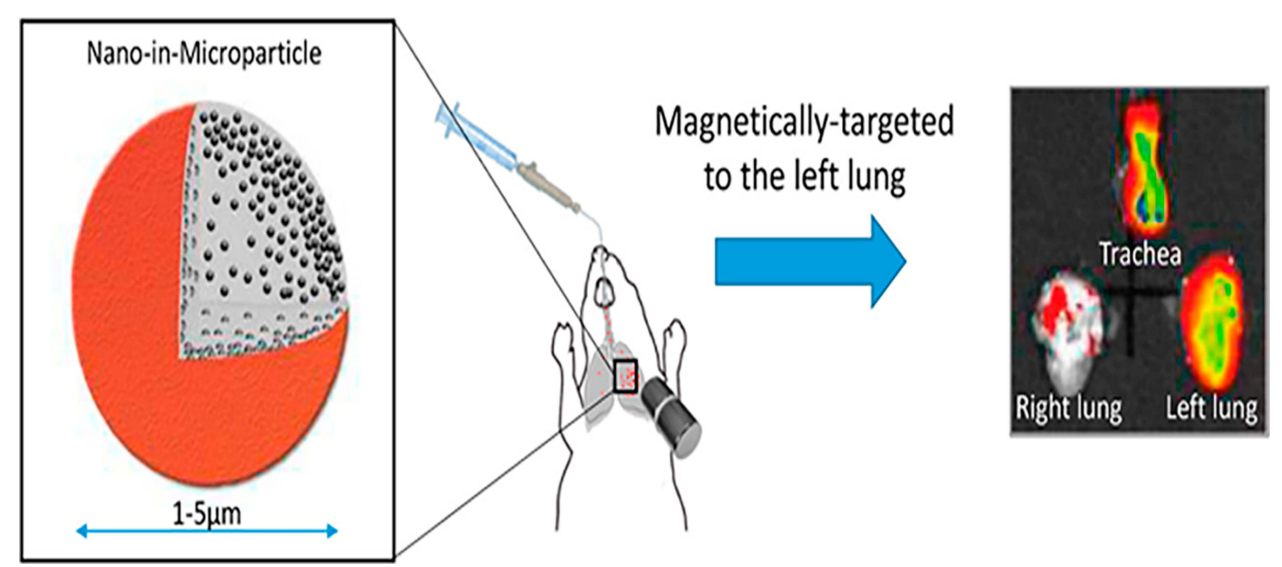

Figure 8 Nano-in microparticles (NIMs) containing superparamagnetic iron oxide nanoparticles (SPIONs) Copyright 20I7, American Chemical Society. ${ }^{130}$

functionalized with negative $\left(-\mathrm{COO}^{-}\right)$or positive $\left(-\mathrm{NH}_{3}{ }^{+}\right)$ surface charges. This was further functionalized with a DCSIGN antibody on the particle surface, that enabled its binding to a dendritic cell surface receptor. Effects of aerosolized AuNPs were assessed using a 3D co-culture model consisting of epithelial and immune cells (macrophages and dendritic cells). It mimicked the epithelial tissue barrier of the human lung and it was inferred that PVA- $\mathrm{NH}_{2}$ AuNPs showed higher uptake and enhanced apoptosis compared to their $-\mathrm{COOH}$ counterparts. These results highlighted the immune engineering approaches for targeting and activation of immune cells in the lungs by nanocarriers ${ }^{140}$ (Figure 9). Cerium oxide-based nanoparticles also emerged as a potential candidate to treat oxidative stress in COPD due to their versatile property to protect cells from ROS. They show SOD and catalase mimetic activity due to their tendency to co-exist either in reduced $(+3)$ or oxidized $(+4)$ state. In +3 oxidized state, they exhibit SOD mimetic activity by catalyzing superoxide radical anion while in +4 reduced state, they show catalase activity by decomposing $\mathrm{H}_{2} \mathrm{O}_{2}$ to $\mathrm{O}_{2}$ and $\mathrm{H}_{2} \mathrm{O}$, thereby protecting the cells from harmful ROS. Recently these nanoparticles have been coupled with protein-based drug delivery systems such as bovine serum albumin, dextran, and silk in order to overcome the disadvantages associated with the use of metal/ metal oxide nanoparticles such as toxicity, immunogenicity, low biodegradability and less biocompatibility. For instance, Bhushan et al successfully delivered cerium oxide nanoparticles by encapsulating it in albumin nanoparticles. ${ }^{141}$ Further, Murlidharan et al successfully designed dimethyl fumarate containing inhalable dry powders that are a first-in-class anti-oxidant $\mathrm{Nrf} 2$ activator used

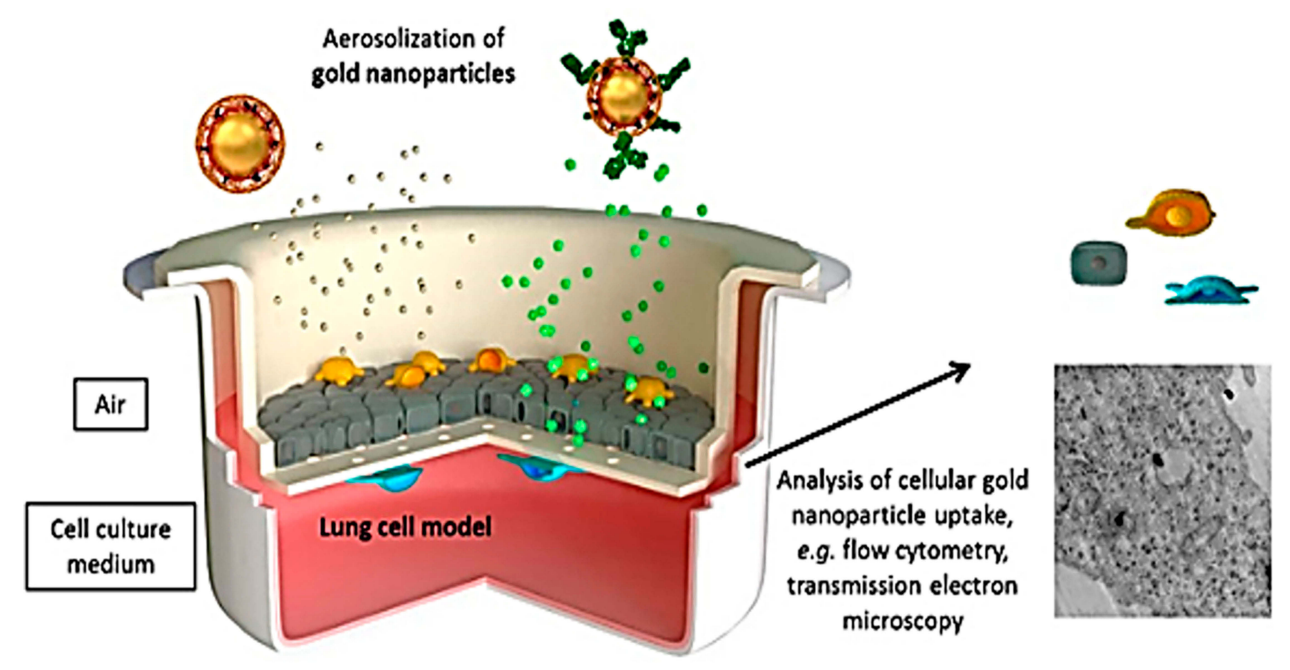

Figure 9 Assessments of fluorescently encoded poly(vinyl alcohol) (PVA)-coated gold nanoparticles using a 3D co-culture model consisting of epithelial and immune cells (macrophages and dendritic cells). Copyright 2016, American Chemical Society. ${ }^{132}$ 
in the treatment of pulmonary inflammation. ${ }^{142}$ The authors predicted that these particles had proficient aerosol deposition patterns. These particles were able to reach the lower airways to treat inflammation in this region in lungs using in vitro predictive lung deposition modelling. Titanium dioxide nanoparticles and carbon black nanoparticles could offer a promising approach for the treatment of COPD as they do not aggravate elastase-induced emphysema as demonstrated by Roulette et al. ${ }^{143}$ However, carbon black nanoparticles induced histologic inflammation and MMP12 mRNA and protein expression in macrophages. ${ }^{143}$ Metallic nanoparticles could also be effectively used as a molecular imaging agent for chronic obstructive lung diseases. Al Faraj et al depicted that the superparamagnetic iron oxide nanoparticles (SPIONs) conjugated with an antibody could be used for noninvasive magnetic resonance imaging (MRI) of macrophage subpopulations. AntiCD86 and anti-CD206 antibody-conjugated SPIONs were administered by intrapulmonary route in LPS-induced mouse model of COPD. Specific affinity to the proinflammatory (M1) and resolutive (M2) macrophage subpopulations was shown by each type of nanoparticle. ${ }^{144}$ Coupling of SPIONs with a targeted antibody to a particular macrophage subpopulation attributes to the selective delivery of nanoparticles to the targeted cells that contributes to high contrast molecular imaging and targeted therapy. ${ }^{144}$ Still, there are major challenges associated with the use of metal/ metal oxide nanoparticles that needs to be addressed such as low drug carrying capacity cargo, toxicity, and use of harmful chemicals used for the formation of these nanoparticles.

\section{Nano-Theranostics for Chronic Obstructive Lung Diseases}

Theranostics involves the combination of diagnosis with therapy and is considered as the future of personalized medicine, which has great potential when it comes to the treatment of chronic inflammatory lung diseases. It will open a new era towards the health care of the lungs. Nano-based theranostics offer many advantages for CF and COPD patients such as realtime diagnosis of the state of lung inflammation and treatment for individual patients with reduced adverse effects of drugs. It consists of developing multifunctional airway targeting nanocarriers, which will fulfil the dual functions of therapy and diagnostics. These nanocarriers will be designed to carry therapeutic agents and they will be simultaneously coupled with imaging moieties, thereby facilitating multiple functions such as cell therapy, targeting, and imaging ultra-sensitivity within a single system. It is critical to address these challenges that we face in treating chronic obstructive lung diseases and thus increase the survival in patients with CF and COPD. ${ }^{133}$

A number of multifunctional metal and metal oxide nanoparticles have been reported for lung cancer such as gold NPs that were used for both enhancing the optical ability and for photothermal cancer therapy. Weng et al developed multifunctional $\mathrm{CdSe} / \mathrm{ZnS}$ quantum dots coupled with liposomes that were used for the targeted delivery of anti-cancer drugs as well as fluorescence imaging agents. ${ }^{145}$ Multifunctional core-shell nanoparticles have also opened a new horizon for theranostic treatment of chronic lung diseases. An example of this is the magnetic iron oxide nanoparticle coupled with the drug and encapsulated silica shell that serves to deliver the payload comprising MRI contrast agent and hydrophobic drug making diagnostics and therapeutics possible in a single system. Despite the recent progress there is a need to develop multifunctional nanosystems for COPD. Moreover, metal oxide nanoparticles developed as cancer theranostic systems have limited applications in chronic airway diseases due to their toxicity and chronic inflammation. ${ }^{146}$ Liposomes have also emerged as a promising material for the fabrication of theranostic hybrids with the ease of encapsulation of quantum dots and multiple drugs (Figure 10). ${ }^{147}$ In addition, amphiphilic block copolymers depending on their relative hydrophobicity/hydrophilicity and chemical structures can form several type of nano-assemblies in aqueous solutions such as vesicles and micelle. These polymeric vesicles are similar to liposomes that can deliver curative agents contained in their hydrophobic membranes and aqueous core. These offer numerous advantages such as controlling physical, biological and chemical properties by modifying the block length, chemistry and functionalization (Figure 10).

\section{Theranostic Nanoparticles for Chronic Obstructive Lung Diseases}

Currently, multifunctional polymeric vesicles synthesised by blending of two synthetic polymers such as poly(ethylene glycol)-poly(lactic-co-glycolic acid) PLGA-PEG are used for delivery of COPD/CF drugs (prednisolone, corticosteroid and/ or anti-inflammatory bronchodilator, theophylline etc.) and molecular probes for theranostic applications in obstructive lung diseases. Such probe and drug-loaded nanocarriers act as a theranostic system for treating $\mathrm{CF}$ and COPD. Various versatile hydrophilic polymers such as PEG, PEI, PEO, PVA have properties such as, high flexibility, low immunogenicity, less toxicity as well as these lack reticuloendothelial system 


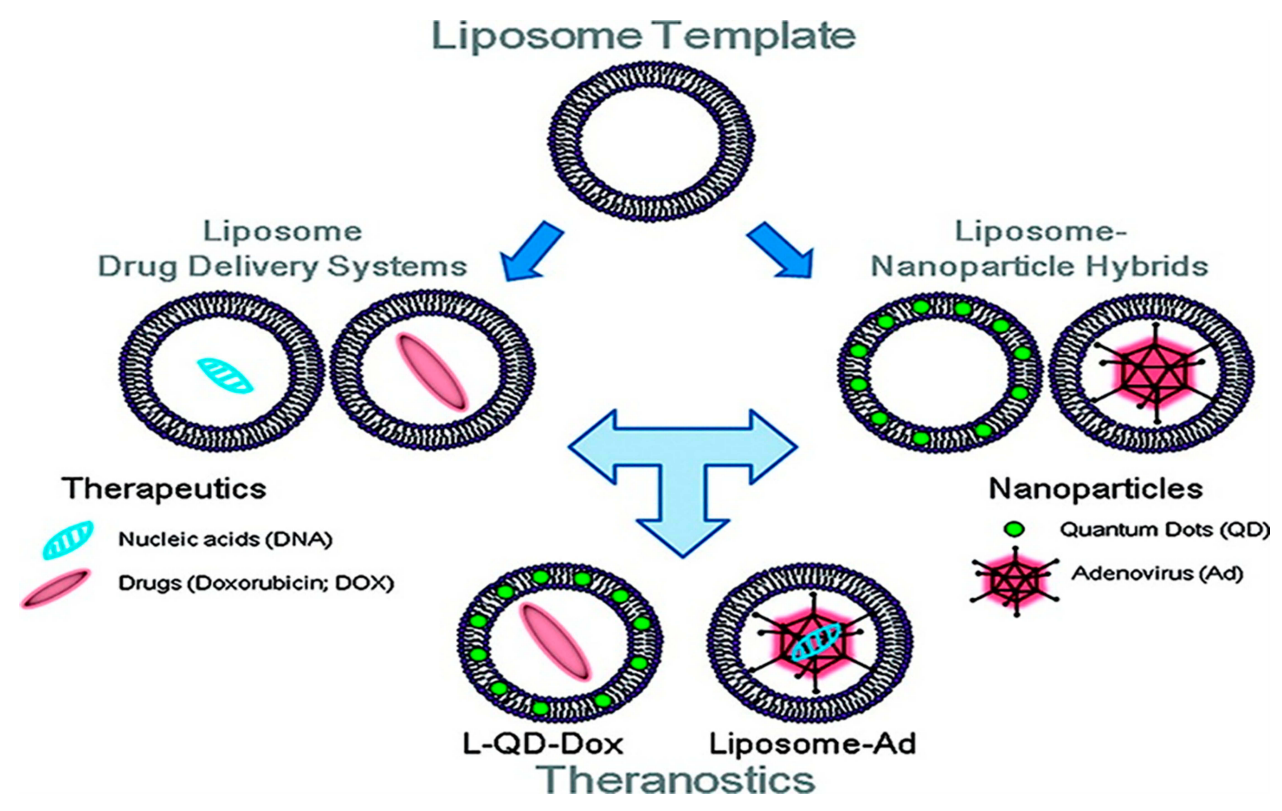

Figure 10 Liposomal-based theranostic nanoparticles. Copyright 20I I, American Chemical Society. ${ }^{85}$

(RES) accumulation that makes the polymer suitable coating agents for nanoparticles in drug delivery applications. PLGA is another bio-degradable and biocompatible co-polymer used in a number of FDA approved therapeutic devices. ${ }^{148}$ Mostly inhaled steroids are used to treat various lung-related diseases like asthma but their pharmacological effect is relatively short. It has been hypnotized that as compared to free steroids positively charged nanoformulations of polymeric steroids accumulate at the site of airway inflammation with higher benefits. For instance, polymeric micelles known as budesonide have shown the ability to reduce inflammatory cell counts in broncho-alveolar region in asthmatic/COPD rat models. Another approach employs liposomes as the nanocarrier to deliver the drug ketofin fumarate which is a mast cell stabilizer to the targeted site in lungs of the rat by dry powder aerosols. ${ }^{149,150}$

Current tools used for monitoring and accessing drug activity are not only time consuming and costly, but also require significant resources for processing. Moreover, the development of such real-time imaging technologies can greatly ameliorate the evaluation of Phase I-III drug development and can reduce the cost of clinical procedures (biopsy, bronchoscopy etc). It is impossible to assess the real-time drug activity in the lung epithelial or inflammatory cells in case of COPD and CF. Moreover, drug-specific responses in the same animal model at different points are currently not feasible. Therefore, in order to monitor both drug development and evaluation of the state of chronic lung diseases in a cost- effective manner, a non-invasive imaging technique is indispensable. The two most extensive techniques that are used for non-invasive and real-time assessment of human lung cancer are single photon emission computed tomography (SPECT/ $\mathrm{CT}$ ) and positron emission tomography (PET/CT). ${ }^{151}$ These involve the detection of positron emitted from radiolabeled tracers that are accumulated at the site of the desired tissue. One such tracer is a radioactive marker FDG (fluorine-18fluorodeoxyglucose). Under inflammatory conditions various cytokines and growth factors increase the affinity for the glucose transporters. FDG accumulates at the site of inflammation mainly in the inflammatory cells (e.g. activated macrophages) making it a desirable probe for inflammatory lung diseases such as COPD and CF. ${ }^{152}$ Thus, the development of novel molecular probes that can detect bacteria/viruses, inflammation, and apoptotic cells that can be more useful for understanding the cellular processes affected in the pathogenesis of chronic obstructive lung diseases.

Gene therapy is another promising approach to treat chronic obstructive lung diseases in addition to antiinflammatory drugs and anti-oxidant therapy. Some of the nanoparticle vectors have successfully achieved significantly higher expression of the transgenes in the lungs. Cationic liposomes have been developed for pulmonary gene delivery. Liposomes that have been conjugated to cell penetrating peptides exhibit enhanced cellular uptake to airway cells. Gene therapy particularly seems to be an attractive approach to treat COPD. It has already shown 
progress with adenoviruses that could partially correct $\mathrm{CF}$ in clinical trials. ${ }^{153}$

\section{Gene Therapy for COPD}

Gene therapy is one of the most promising strategies for the treatment of chronic lung diseases such as COPD that is progressive in nature. The first step in the treatment of COPD involves identification of the defective gene. Once it is identified, the next step would be the vector design and delivery. Adenoviral vector-based gene therapy has been regarded as a favourable strategy for lung diseases. ${ }^{154}$ Viral liposome complexes carrying the AAT ( $\alpha 1$ anti-trypsin) gene in mice produced detectable levels of AAT in the serum for up to 30 days on intravenous administration. AAVs (adeno-associated vectors) have been other promising vectors to deliver AAT transgene with a high level of transgene expression, low toxicity and longer duration. Lipoplexes also have been known to transfer AAT transgene using intranasal transfer with similar success to that of non-viral vectors, but they face several limitations while administration. RNA/DNA oligonucleotides directed repair for site-specific correction of single bases carried in chromosomes, known as genoplasty, is the new technology under development at present. However, its potential for AAT transfer still remains to be investigated. ${ }^{155,156,157}$

\section{Stem Cell Therapy for COPD}

Recent advances in the stem cell research related to bone marrow mesenchymal stem cells (BM-MSCs) have provided a novel approach into the treatment of chronic pulmonary diseases such as COPD. It has been reported that in rat models of LPS and CS-induced lung injury MSCs differentiate into type I and type II alveolar epithelial cells. Liu et al have shown the differentiation of MSCs in a co-culture system into type II alveolar epithelial cells through the activation of the canonical Wnt signalling pathway. Moreover, after the transplantation of BMMSCs, a decrease in the oxidative stress was accompanying the increased survival rate of LPS-induced lung injury in rats. MSCs also decreased the oxidative burden in the brain of rat model in case of spontaneous stroke suggesting that MSCs may also reduce the oxidative stress in CSinduced emphysema. There was a suppression of alveolar apoptosis in COPD rat models by MSCs transplantation due to the stimulation of vascular endothelial growth factor (VEGF) secretion. ${ }^{158}$ MSC transplantation offers a potentially promising treatment strategy of COPD and involves the modulation of inflammation, apoptosis, oxidative stress and protease/anti-protease balance. The dearth of data and long-term safety of MSCs in COPD patients is one of the major obstacles to clinical applications of MSCs. ${ }^{159}$

Although the clinical trials with MSCs have shown no infusion toxicity or attributed to the death of treated patient during 2-year follow-up large scale clinical trials are still required to thoroughly assess the efficacy and long-term safety to the use of MSCs. Apart from this, another challenge in MSC therapy is that its therapeutic schedule remain unclear and additional studies are required to ascertain the appropriate dosage, route of administration and infusion rate. The final challenge associated with MSCs is the low level of engraftment in host organs and their poor survival. ${ }^{160}$ Therefore, new approaches need to be developed to increase the survival rate of MSCs in host organs. Despite the challenges, MSC therapy is one of the promising approaches for COPD therapy.

\section{Conclusion and future directions}

At present, there are no therapies that could completely cure the pathogenesis of COPD. However, the advent of nanotechnology has offered scope to improve the existing treatment strategies of COPD including the anti-oxidant pharmacological therapies. A variety of nanocarriers have the ability to improve efficiency and decrease the toxicity for a wide variety of drugs. Advances in the field of nanotechnology have resulted in the development of novel therapeutic approaches to treat various chronic lung disorders using nanocarriers and their modification in surfaces. Targeting ligands are used to allow the development of smart multifunctional drug carriers. However, this technology needs to be fully developed and hurdles need to be rectified before we realize its full clinical potential. Translating the "smart technology" into the clinical purpose, the ratio of cost/benefit needs to be considered, for example; requirement and identification of a large number of targeting ligands and understanding the toxicity of nanoparticles and their effects on the immune system. There is a need to fabricate novel nanomaterials that exhibit lung clearance mechanisms also after being deposited on the lungs. Combining gene and stem cell therapy approaches with nanotechnology can offer exemplary scope for the development of effective strategies in COPD and other chronic airway disease therapeutics.

\section{Acknowledgments}

IKS is supported by the R01 HL142543 from the National Heart, Lung, and Blood Institute (NHLBI), National 
Institutes of Health, USA. GP acknowledge the financial support of the Department of Science and Technology (DST)-International Division (grant number: INT/RUS/ RFBR/P-235), Government of India. SS is thankful to the Department of Biotechnology, Government of India, for providing the fellowship. M.P. and S.C are thankful to the Ministry of Human Resource Development, Government of India, for the fellowship.

\section{Disclosure}

The authors report no conflicts of interest in this work.

\section{References}

1. Yao H, Rahman I. Current concepts on oxidative/carbonyl stress, inflammation and epigenetics in pathogenesis of chronic obstructive pulmonary disease. Toxicol Appl Pharmacol. 2011;254:7285. doi:10.1016/j.taap.2009.10.022

2. Thorley AJ, Tetley TD. Pulmonary epithelium, cigarette smoke, and chronic obstructive pulmonary disease. Int J Chron Obstruct Pulmon Dis. 2007;2:409-428.

3. van Eeden SF, Sin DD. Oxidative stress in chronic obstructive pulmonary disease: a lung and systemic process. Can Respir J. 2013;20:27-29. doi:10.1155/2013/509130

4. Silva-Palacios A, Ostolga-Chavarría M, Sánchez-Garibay C, et al. Sulforaphane protects from myocardial ischemia-reperfusion damage through the balanced activation of Nrf2/AhR. Free Radic Biol Med. 2019;143:331-340. doi:10.1016/j.freeradbiomed. 2019.08.012

5. Lin X, Bai D, Wei Z, et al. Curcumin attenuates oxidative stress in RAW264.7 cells by increasing the activity of antioxidant enzymes and activating the Nrf2-Keap1 pathway. PLoS One. 2019;14:e0216711. doi:10.1371/journal.pone.0216711

6. Kumar H, Kim IS, More SV, Kim BW, Choi DK. Natural product-derived pharmacological modulators of Nrf2/ARE pathway for chronic diseases. Nat Prod Rep. 2014;31:109-139.

7. Rahman I, Macnee W. Antioxidant pharmacological therapies for COPD . Curr Opin Pharmacol. 2012;12:256-265.

8. Rahman I. Antioxidant therapies in COPD. Int J Chron Obstruct Pulmon Dis. 2006;1:15-29. doi:10.2147/copd.2006.1.1.15

9. Sadowska AM, Verbraecken J, Darquennes K, De Backer WA. Role of $\mathrm{N}$-acetylcysteine in the management of COPD. Int $J$ Chron Obstruct Pulmon Dis. 2006;1:425-434. doi:10.2147/ copd.2006.1.4.425

10. Dodd S, Dean O, Copolov DL, Malhi GS, Berk M. $N$-acetylcysteine for antioxidant therapy: pharmacology and clinical utility. Expert Opin Biol Ther. 2008;8:1955-1962. doi:10.1517/147282 20802517901

11. Burgunder JM, Varriale A, Lauterburg BH. Effect of $\mathrm{N}$-acetylcysteine on plasma cysteine and glutathione following paracetamol administration. Eur J Clin Pharmacol. 1989;36:127131. doi:10.1007/BF00609183

12. Rubio ML, Martin-Mosquero MC, Ortega M, Peces-Barba G, González-Mangado N. Oral N-acetylcysteine attenuates elastaseinduced pulmonary emphysema in rats. Chest. 2004;125:15001506. doi:10.1378/chest.125.4.1500

13. Cazzola M, Calzetta L, Facciolo F, Rogliani P, Matera MG. Pharmacological investigation on the anti-oxidant and antiinflammatory activity of $\mathrm{N}$-acetylcysteine in an ex vivo model of COPD exacerbation. Respir Res. 2017;18:26. doi:10.1186/ s12931-016-0500-y
14. Adler KB, Hendley DD, Davis GS. Bacteria associated with obstructive pulmonary disease elaborate extracellular products that stimulate mucin secretion by explants of guinea pig airways. Am J Pathol. 1986;125:501-514.

15. Besaratinia A, Besarati Nia A, Maas LM, et al. Applicability of induced sputum for molecular dosimetry of exposure to inhalatory carcinogens: 32P-postlabeling of lipophilic DNA adducts in smokers and nonsmokers. Cancer Epidemiol Biomarkers Prev. 2000;9:367-372.

16. De Benedetto F, Aceto A, Dragani B, et al. Long-term oral n-acetylcysteine reduces exhaled hydrogen peroxide in stable COPD. Pulm Pharmacol Ther. 2005;18:41-47. doi:10.1016/j. pupt.2004.09.030

17. Aylward M, Maddock J, Dewland P. Clinical evaluation of acetylcysteine in the treatment of patients with chronic obstructive bronchitis: a balanced double-blind trial with placebo control. Eur J Respir Dis Suppl. 1980;111:81-89.

18. Stey C, Steurer J, Bachmann S, Medici TC, Tramèr MR. The effect of oral N-acetylcysteine in chronic bronchitis: a quantitative systematic review. Eur Respir J. 2000;16:253-262. doi:10. 1034/j.1399-3003.2000.16b12.x

19. Lee KS, Kim SR, Park HS, et al. A novel thiol compound, $\mathrm{N}$-acetylcysteine amide, attenuates allergic airway disease by regulating activation of NF- $\mathrm{KB}$ and hypoxia-inducible factor- $1 \alpha$. Exp Mol Med. 2007;39:756-768. doi:10.1038/emm.2007.82

20. Koechlin C, Couillard A, Cristol JP, et al. Does systemic inflammation trigger local exercise-induced oxidative stress in COPD? Eur Respir J. 2004;23:538-544. doi:10.1183/09031936.04.000 69004

21. Dueholm M, Nielsen C, Thorshauge H, et al. N-acetylcysteine by metered dose inhaler in the treatment of chronic bronchitis: a multi-centre study. Respir Med. 1992;86:89-92. doi:10.1016/ S0954-6111(06)80220-9

22. Antonicelli F, Parmentier M, Drost EM, et al. Nacystelyn inhibits oxidant-mediated interleukin-8 expression and NF-kappaB nuclear binding in alveolar epithelial cells. Free Radic Biol Med. 2002;32:492-502. doi:10.1016/S0891-5849(01)00820-6

23. Antonicelli F, Brown D, Parmentier M, et al. Regulation of LPSmediated inflammation in vivo and in vitro by the thiol antioxidant Nacystelyn. Am J Physiol Cell Mol Physiol. 2004;286: L1319-L1327. doi:10.1152/ajplung.00329.2003

24. Gillissen A, Jaworska M, Orth M, et al. Nacystelyn, a novel lysine salt of $\mathrm{N}$-acetylcysteine, to augment cellular antioxidant defence in vitro. Respir Med. 1997;91:159-168. doi:10.1016/ S0954-6111(97)90052-4

25. Elborn JS, Bell SC, Madge SL, et al. Report of the European Respiratory Society/European Cystic Fibrosis Society task force on the care of adults with cystic fibrosis. Eur Respir J. 2016;47:420-428. doi:10.1183/13993003.00592-2015

26. Ekberg-Jansson A, Larson M, MacNee W, et al. N-isobutyrylcysteine, a donor of systemic thiols, does not reduce the exacerbation rate in chronic bronchitis. Eur Respir J. 1999;13:829-834. doi:10.1034/j.1399-3003.1999.13d22.x

27. Macciò A, Madeddu C, Panzone F, Mantovani G. Carbocysteine: clinical experience and new perspectives in the treatment of chronic inflammatory diseases. Expert Opin Pharmacother. 2009;10:693-703. doi:10.1517/14656560902758343

28. Braga PC, Allegra L, Rampoldi C, Ornaghi A, Beghi G. Longlasting effects on rheology and clearance of bronchial mucus after short-term administration of high doses of carbocysteine-lysine to patients with chronic bronchitis. Respiration. 1990;57:353-358. doi:10.1159/000195871

29. Yasuo M, Fujimoto K, Imamura H, et al. 1-Carbocisteine reduces neutrophil elastase-induced mucin production. Respir Physiol Neurobiol. 2009;167:214-216. doi:10.1016/j.resp.2009.04.016 
30. Yoshida M, Nakayama K, Yasuda H, et al. Carbocisteine inhibits oxidant-induced apoptosis in cultured human airway epithelial cells. Respirology. 2009;14:1027-1034. doi:10.1111/j.14401843.2009.01594.x

31. Pace E, Ferraro M, Siena L, et al. Carbocysteine regulates innate immune responses and senescence processes in cigarette smoke stimulated bronchial epithelial cells. Toxicol Lett. 2013;223:198204. doi:10.1016/j.toxlet.2013.09.013

32. Wang W, Zheng J-P, Zhu S-X, et al. Carbocisteine attenuates hydrogen peroxide-induced inflammatory injury in A549 cells via NF- $\kappa$ B and ERK1/2 MAPK pathways. Int Immunopharmacol. 2015;24:306-313. doi:10.1016/j.intimp.2014.12.018

33. Hooper C, Calvert J. The role for S-carboxymethylcysteine (carbocisteine) in the management of chronic obstructive pulmonary disease. Int J COPD. 2008;3:659-669. doi:10.2147/COPD.S3735

34. Zheng CH, Ahmed K, Rikitomi N, Martinez G, Nagatake T. The effects of S-carboxymethylcysteine and $\mathrm{N}$-acetylcysteine on the adherence of Moraxella catarrhalis to human pharyngeal epithelial cells. Microbiol Immunol. 1999;43:107-113. doi:10.1111/ j.1348-0421.1999.tb02381.x

35. Cakan G, Turkoz M, Turan T, Ahmed K, Nagatake T. S-carboxymethylcysteine inhibits the attachment of Streptococcus pneumoniae to human pharyngeal epithelial cells. Microb Pathog. 2003;34:261-265. doi:10.1016/S0882-4010(03)00048-2

36. Moretti M, Fagnani S. Erdosteine reduces inflammation and time to first exacerbation postdischarge in hospitalized patients with AECOPD. Int J Chron Obstruct Pulmon Dis. 2015;10:23192325. doi:10.2147/COPD.S87091

37. Moretti M, Bottrighi P, Dallari R, et al. The effect of long-term treatment with erdosteine on chronic obstructive pulmonary disease: the EQUALIFE Study. Drugs Exp Clin Res. 2004;30:143-152.

38. Moretti M. Erdosteine: its relevance in COPD treatment. Expert Opin Drug Metab Toxicol. 2009;5:333-343. doi:10.1517/1742 5250902814790

39. Dal Negro, R.W., Wedzicha, J.A., Iversen, M., Fontana, G., Page, C., Cicero, A.F., Pozzi, E. and Calverley, P.M., 2017. Effect of erdosteine on the rate and duration of COPD exacerbations: the RESTORE study. Eur Respir J. 50:1700711

40. Cazzola M, Floriani I, Page CP. The therapeutic efficacy of erdosteine in the treatment of chronic obstructive bronchitis: a meta-analysis of individual patient data. Pulm Pharmacol Ther. 2010;23:135-144. doi:10.1016/j.pupt.2009.10.002

41. Negro RWD. Erdosteine: antitussive and anti-inflammatory effects. Lung. 2008;186:70-73. doi:10.1007/s00408-007-9065-3

42. Dal Negro RW, Visconti M, Tognella S, Micheletto C. Erdosteine affects eicosanoid production in COPD. Int $J$ Clin Pharmacol Ther. 2011;49:41-45. doi:10.5414/CPP49041

43. Komatsu H, Yamaguchi S, Komorita N, et al. Inhibition of endotoxin- and antigen-induced airway inflammation by fudosteine, a mucoactive agent. Pulm Pharmacol Ther. 2005;18:121-127. doi:10.1016/j.pupt.2004.11.002

44. Rhee CK, Kang CM, You MB, et al. Effect of fudosteine on mucin production. Eur Respir J. 2008;32:1195-1202. doi:10.1183/09031936.00018508

45. Osoata GO, Hanazawa T, Brindicci C, et al. Peroxynitrite elevation in exhaled breath condensate of COPD and its inhibition by fudosteine. Chest. 2009;135:1513-1520. doi:10.1378/chest.082105

46. Hodge S, Matthews G, Mukaro V, et al. Cigarette smoke-induced changes to alveolar macrophage phenotype and function are improved by treatment with procysteine. Am J Respir Cell Mol Biol. 2011;44:673-681. doi:10.1165/rcmb.2009-0459OC

47. Kensler TW, Wakabayashi N, Biswal S. Cell survival responses to environmental stresses via the Keap1-Nrf2-ARE pathway. Annu Rev Pharmacol Toxicol. 2007;47:89-116. doi:10.1146/annurev. pharmtox.46.120604.141046
48. Sykiotis GP, Bohmann D. Stress-activated cap'n'collar transcription factors in aging and human disease. Sci Signal. 2010;3:re3. doi:10.1126/scisignal.3112re3

49. Itoh K, Mimura J, Yamamoto M. Discovery of the negative regulator of Nrf2, Keap1: a historical overview. Antioxid Redox Signal. 2010;13:1665-1678. doi:10.1089/ars.2010.3222

50. Clements CM, McNally RS, Conti BJ, Mak TW, Ting JP-Y. DJ-1, a cancer- and Parkinson's disease-associated protein, stabilizes the antioxidant transcriptional master regulator Nrf2. Proc Natl Acad Sci. 2006;103:15091-15096. doi:10.1073/pnas.0607260103

51. Kaspar JW, Niture SK, Jaiswal AK. Nrf2:INrf2 (Keap1) signaling in oxidative stress. Free Radic Biol Med. 2009;47:1304-1309. doi:10.1016/j.freeradbiomed.2009.07.035

52. Plafker KS, Nguyen L, Barneche M, et al. The ubiquitin-conjugating enzyme UbcM2 can regulate the stability and activity of the antioxidant transcription factor Nrf2. J Biol Chem. 2010;285:23064-23074. doi:10.1074/jbc.M110.121913

53. Keum Y-S, Yu S, Chang PP-J, et al. Mechanism of action of sulforaphane: inhibition of p38 mitogen-activated protein kinase isoforms contributing to the induction of antioxidant response element-mediated heme oxygenase-1 in human hepatoma HepG2 cells. Cancer Res. 2006;66:8804-8813. doi:10.1158/ 0008-5472.CAN-05-3513

54. Yoon H-Y, Kang N-I, Lee H-K, et al. Sulforaphane protects kidneys against ischemia-reperfusion injury through induction of the Nrf2-dependent phase 2 enzyme. Biochem Pharmacol. 2008;75:2214-2223. doi:10.1016/j.bcp.2008.02.029

55. Suzuki M, Betsuyaku T, Ito Y, et al. Curcumin attenuates elastaseand cigarette smoke-induced pulmonary emphysema in mice. Am J Physiol Cell Mol Physiol. 2009;296:L614-L623. doi:10.1152/ ajplung.90443.2008

56. Chen C-Y, Jang J-H, Li M-H, Surh Y-J. Resveratrol upregulates heme oxygenase-1 expression via activation of NF-E2-related factor 2 in PC12 cells. Biochem Biophys Res Commun. 2005;331:993-1000. doi:10.1016/j.bbrc.2005.03.237

57. Kode A, Rajendrasozhan S, Caito S, et al. Resveratrol induces glutathione synthesis by activation of $\mathrm{Nrf} 2$ and protects against cigarette smoke-mediated oxidative stress in human lung epithelial cells. Am J Physiol Cell Mol Physiol. 2008;294:L478-L488. doi:10.1152/ajplung.00361.2007

58. Andreadi CK, Howells LM, Atherfold PA, Manson MM Involvement of Nrf2, p38, B-raf and NF- B, but not PI3K, in induction of hemeoxygenase (HO-1) by dietary polyphenols. $\mathrm{Mol}$ Pharmacol. 2005;69:1033-1040. doi:10.1124/mol.105.018374

59. Shah ZA, Li R-C, Ahmad AS, et al. The flavanol (-)-Epicatechin prevents stroke damage through the Nrf2/HO1 pathway. J Cereb Blood Flow Metab. 2010;30:1951-1961. doi:10.1038/jcbfm. 2010.53

60. Sussan TE, Rangasamy T, Blake DJ, et al. Targeting Nrf2 with the triterpenoid CDDO- imidazolide attenuates cigarette smokeinduced emphysema and cardiac dysfunction in mice. Proc Natl Acad Sci. 2009;106:250-255. doi:10.1073/pnas.0804333106

61. Ichikawa T, Li J, Meyer CJ, et al. Dihydro-CDDO-trifluoroethyl amide (dh404), a Novel Nrf2 activator, suppresses oxidative stress in cardiomyocytes. PLoS One. 2009;4:e8391. doi:10.1371/ journal.pone.0008391

62. Hsu C-L, Wu Y-L, Tang G-J, Lee T-S, Kou YR. Ginkgo biloba extract confers protection from cigarette smoke extract-induced apoptosis in human lung endothelial cells: role of heme oxygenase-1. Pulm Pharmacol Ther. 2009;22:286-296. doi:10.1016/j. pupt.2009.02.003

63. Yao P, Nussler A, Liu L, et al. Quercetin protects human hepatocytes from ethanol-derived oxidative stress by inducing heme oxygenase1 via the MAPK/Nrf2 pathways. J Hepatol. 2007;47:253-261. doi:10.1016/j.jhep.2007.02.008 
64. Rushworth SA, Ogborne RM, Charalambos CA, O'Connell MA. Role of protein kinase $\mathrm{C} \delta$ in curcumin-induced antioxidant response element-mediated gene expression in human monocytes. Biochem Biophys Res Commun. 2006;341:1007-1016. doi:10.10 16/j.bbrc.2006.01.065

65. Muscoli C, Sacco I, Alecce W, et al. The protective effect of superoxide dismutase mimetic M40401 on balloon injury-related neointima formation: role of the lectin-like oxidized low-density lipoprotein receptor-1. J Pharmacol Exp Ther. 2004;311:44-50. doi:10.1124/jpet.104.068205

66. Tuder RM, Zhen L, Cho CY, et al. Oxidative stress and apoptosis interact and cause emphysema due to vascular endothelial growth factor receptor blockade. Am J Respir Cell Mol biol. 2003;29:8897. doi:10.1165/rcmb.2002-02280C

67. Chang L-Y, Crapo JD. Inhibition of airway inflammation and hyperreactivity by an antioxidant mimetic. Free Radic Biol Med. 2002;33:379-386. doi:10.1016/S0891-5849(02)00919-X

68. Smith KR, Uyeminami DL, Kodavanti UP, et al. Inhibition of tobacco smoke-induced lung inflammation by a catalytic antioxidant. Free Radic Biol Med. 2002;33:1106-1114. doi:10.1016/ S0891-5849(02)01003-1

69. Sharpe MA, Ollosson R, Stewart VC, Clark JB. Oxidation of nitric oxide by oxomanganese-salen complexes: a new mechanism for cellular protection by superoxide dismutase/catalase mimetics. Biochem J. 2002;366:97-107. doi:10.1042/bj20020154

70. Kinnula VL, Crapo JD. Superoxide dismutases in the lung and human lung diseases. Am $J$ Respir Crit Care Med. 2003;167:1600-1619. doi:10.1164/rccm.200212-1479SO

71. Yao H, Arunachalam G, Hwang J-W, et al. Extracellular superoxide dismutase protects against pulmonary emphysema by attenuating oxidative fragmentation of ECM. Proc Natl Acad Sci. 2010;107:15571-15576. doi:10.1073/pnas.1007625107

72. Tollefson AK, Oberley-Deegan RE, Butterfield KT, et al. Endogenous enzymes (NOX and ECSOD) regulate smokeinduced oxidative stress. Free Radic Biol Med. 2010;49:19371946. doi:10.1016/j.freeradbiomed.2010.09.022

73. József L, Filep JG. Selenium-containing compounds attenuate peroxynitrite-mediated NF-kappaB and AP-1 activation and interleukin-8 gene and protein expression in human leukocytes. Free Radic Biol Med. 2003;35:1018-1027. doi:10.1016/S0891-5849 (03)00439-8

74. Zhang M, Nomura A, Uchida Y, et al. Ebselen suppresses late airway responses and airway inflammation in guinea pigs. Free Radic Biol Med. 2002;32:454-464. doi:10.1016/S0891-5849(01)00825-5

75. Haddad E-B, McCluskie K, Birrell MA, et al. Differential effects of ebselen on neutrophil recruitment, chemokine, and inflammatory mediator expression in a rat model of lipopolysaccharideinduced pulmonary inflammation. J Immunol. 2002;169:974-982. doi:10.4049/jimmunol.169.2.974

76. Chabrier PE, Auguet M, Spinnewyn B, et al. BN 80933, a dual inhibitor of neuronal nitric oxide synthase and lipid peroxidation: a promising neuroprotective strategy. Proc Natl Acad Sci U S A. 1999;96:10824-10829. doi:10.1073/pnas.96.19.10824

77. Yhee JY, Im J, Nho RS. Advanced therapeutic strategies for chronic lung disease using nanoparticle-based drug delivery. $J$ Clin Med. 2016;5:82. doi:10.3390/jcm5090082

78. Jiang J, Oberdörster G, Biswas P. Characterization of size, surface charge, and agglomeration state of nanoparticle dispersions for toxicological studies. Journal of Nanoparticle Research. 2009;11 (1):77-89. doi:10.1007/s11051-008-9446-4

79. Janib SM, Moses AS, MacKay JA. Imaging and drug delivery using theranostic nanoparticles. Adv Drug Deliv Rev. 2010;62:1052-1063. doi:10.1016/j.addr.2010.08.004

80. van Rijt SH, Bein T, Meiners S. Medical nanoparticles for next generation drug delivery to the lungs. Eur Respir J. 2014;44:765774. doi:10.1183/09031936.00212813
81. Suk JS, Boylan NJ, Trehan K, et al. N-acetylcysteine enhances cystic fibrosis sputum penetration and airway gene transfer by highly compacted DNA nanoparticles. Mol Ther. 2011;19:19811989. doi:10.1038/mt.2011.160

82. Rytting E, Nguyen J, Wang X, Kissel T. Biodegradable polymeric nanocarriers for pulmonary drug delivery. Expert Opin Drug Deliv. 2008;5:629-639. doi:10.1517/17425247.5.6.629

83. Sanders N, Rudolph C, Braeckmans K, De Smedt SC, Demeester J. Extracellular barriers in respiratory gene therapy. Adv Drug Deliv Rev. 2009;61:115-127. doi:10.1016/j.addr.2008.09.011

84. Lai SK, Wang -Y-Y, Hanes J. Mucus-penetrating nanoparticles for drug and gene delivery to mucosal tissues. Adv Drug Deliv Rev. 2009;61:158-171. doi:10.1016/j.addr.2008.11.002

85. Geiser M, Quaile O, Wenk A, et al. Cellular uptake and localization of inhaled gold nanoparticles in lungs of mice with chronic obstructive pulmonary disease. Part Fibre Toxicol. 2013;10:19. doi:10.1186/1743-8977-10-19

86. Geiser M, Stoeger T, Casaulta M, Chen S, Semmler-Behnke M, Bolle I, Takenaka S, Kreyling WG, Schulz H. Biokinetics of nanoparticles and susceptibility to particulate exposure in a murine model of cystic fibrosis. Particle and fibre toxicology. 2014 Dec;11(1):19.

87. Blanco E, Shen H, Ferrari M. Principles of nanoparticle design for overcoming biological barriers to drug delivery. Nat Biotechnol. 2015;33:941-951. doi:10.1038/nbt.3330

88. Garbuzenko O, Mainelis G, Taratula O, Minko T. Inhalation treatment of lung cancer: the influence of composition, size and shape of nanocarriers on their lung accumulation and retention. Cancer Biol Med. 2014;11:44

89. Geng Y, Dalhaimer P, Cai S, et al. Shape effects of filaments versus spherical particles in flow and drug delivery. Nat Nanotechnol. 2007;2:249-255. doi:10.1038/nnano.2007.70

90. Champion JA, Mitragotri S. Role of target geometry in phagocytosis. Proc Natl Acad Sci. 2006;103:4930-4934. doi:10.1073/ pnas.0600997103

91. Champion JA, Mitragotri S. Shape induced inhibition of phagocytosis of polymer particles. Pharm Res. 2009;26:244-249. doi:10.1007/s11095-008-9626-Z

92. Alexis F, Pridgen E, Molnar LK, Farokhzad OC. Factors affecting the clearance and biodistribution of polymeric nanoparticles. $\mathrm{Mol}$ Pharm. 2008;5:505-515. doi:10.1021/mp800051m

93. Nel AE, Mädler L, Velegol D, et al. Understanding biophysicochemical interactions at the nano-bio interface. Nat Mater. 2009;8:543-557. doi:10.1038/nmat2442

94. Schütz CA, Juillerat-Jeanneret L, Mueller H, et al. Therapeutic nanoparticles in clinics and under clinical evaluation. Nanomedicine. 2013;8:449-467. doi:10.2217/nnm.13.8

95. Oh YJ, Lee J, Seo JY, et al. Preparation of budesonide-loaded porous PLGA microparticles and their therapeutic efficacy in a murine asthma model. J Control Release. 2011;150:56-62. doi:10.1016/j.jconrel.2010.11.001

96. Taratula O, Garbuzenko OB, Chen AM, Minko T. Innovative strategy for treatment of lung cancer: targeted nanotechnology-based inhalation co-delivery of anticancer drugs and siRNA. J Drug Target. 2011;19:900-914. doi:10.3109/1061186X.2011.622404

97. Trapani. A, Gioia S, Castellani S, et al. Nanocarriers for respiratory diseases treatment: recent advances and current challenges. Curr Top Med Chem. 2014;14:1133-1147. doi:10.2174/ 1568026614666140329225817

98. da Silva AL, Cruz FF, Rocco PRM, Morales MM. New perspectives in nanotherapeutics for chronic respiratory diseases. Biophys Rev. 2017;9:793-803. doi:10.1007/s12551-017-0319-x

99. Gorain B, Choudhury H, Pandey M, et al. Dendrimer-based nanocarriers in lung Cancer therapy. In Nanotechnology-Based Targeted Drug Delivery Systems for Lung Cancer. Academic Press; 2019:161-192. 
100. Zahoor A, Sharma S, Khuller GK, Khuller GK. Inhalable alginate nanoparticles as antitubercular drug carriers against experimental tuberculosis. Int $J$ Antimicrob Agents. 2005;26:298-303. doi:10.1016/j.ijantimicag.2005.07.012

101. Zhang L, Gu FX, Chan JM, et al. Nanoparticles in medicine: therapeutic applications and developments. Clin Pharmacol Ther. 2008;83:761-769. doi:10.1038/sj.clpt.6100400

102. Yoo J-W, Doshi N, Mitragotri S. Adaptive micro and nanoparticles: temporal control over carrier properties to facilitate drug delivery. Adv Drug Deliv Rev. 2011;63:1247-1256. doi:10.1016/j. addr.2011.05.004

103. Amoozgar Z, Yeo Y. Recent advances in stealth coating of nanoparticle drug delivery systems. Wiley Interdiscip Rev Nanomed Nanobiotechnol. 2012;4:219-233. doi:10.1002/wnan.1157

104. Doh K-O, Yeo Y. Application of polysaccharides for surface modification of nanomedicines. Ther Deliv. 2012;3:1447-1456. doi:10.4155/tde. 12.105

105. Monopoli MP, Åberg C, Salvati A, Dawson KA. Biomolecular coronas provide the biological identity of nanosized materials. Nat Nanotechnol. 2012;7:779-786. doi:10.1038/nnano.2012.207

106. Ruge CA, Schaefer UF, Herrmann J, et al. The interplay of lung surfactant proteins and lipids assimilates the macrophage clearance of nanoparticles. PLoS One. 2012;7:e40775. doi:10.1371/ journal.pone.0040775

107. Kumar P, Bohidar HB. Interaction of soot derived multi-carbon nanoparticles with lung surfactants and their possible internalization inside alveolar cavity. Indian J Exp Biol. 2010;48:10371042 .

108. Hashizume H, Baluk P, Morikawa S, et al. Openings between defective endothelial cells explain tumor vessel leakiness. Am J Pathol. 2000;156:1363-1380. doi:10.1016/S0002-9440(10) 65006-7

109. Mehra NK, Mishra V, Jain NK. Receptor-based targeting of therapeutics. Ther Deliv. 2013;4:369-394. doi:10.4155/tde.13.6

110. Yoo HS, Park TG. Folate receptor targeted biodegradable polymeric doxorubicin micelles. J Control Release. 2004;96:273-283. doi:10.1016/j.jconrel.2004.02.003

111. Daniels TR, Bernabeu E, Rodríguez JA, et al. The transferrin receptor and the targeted delivery of therapeutic agents against cancer. Biochim Biophys Acta - Gen Subj. 2012;1820:291-317. doi:10.1016/j.bbagen.2011.07.016

112. Wiewrodt R, Thomas AP, Cipelletti L, et al. Size-dependent intracellular immunotargeting of therapeutic cargoes into endothelial cells. Blood. 2002;99:912-922. doi:10.1182/blood. V99.3.912

113. Meyer M, Wagner E. pH-responsive shielding of non-viral gene vectors. Expert Opin Drug Deliv. 2006;3:563-571. doi:10.1517/ 17425247.3.5.563

114. Hatakeyama H, Akita H, Kogure K, et al. Development of a novel systemic gene delivery system for cancer therapy with a tumorspecific cleavable PEG-lipid. Gene Ther. 2007;14:68-77. doi:10.1038/sj.gt.3302843

115. Geers B, Dewitte H, De Smedt SC, Lentacker I. Crucial factors and emerging concepts in ultrasound-triggered drug delivery. $J$ Control Release. 2012;164:248-255. doi:10.1016/j.jconrel.2012. 08.014

116. Wang X, Liu P, Yang W, et al. Microbubbles coupled to methotrexate-loaded liposomes for ultrasound-mediated delivery of methotrexate across the blood-brain barrier. Int $J$ Nanomedicine. 2014;9:4899-4909. doi:10.2147/IJN.S69845

117. Kuzmov A, Minko T. Nanotechnology approaches for inhalation treatment of lung diseases. J Control Release. 2015;219:500-518. doi:10.1016/j.jconrel.2015.07.024

118. Levchenko TS, Hartner WC, Torchilin VP. Liposomes in diagnosis and treatment of cardiovascular disorders. Methodist Debakey Cardiovasc J. 2012;8:36-41. doi:10.14797/mdcj-8-1-36
119. Pattni BS, Chupin VV, Torchilin VP. New developments in liposomal drug delivery. Chem.Rev. 2015;115:10938-10966. doi:10.1021/acs.chemrev.5b00046

120. Willis L, Hayes D, Mansour HM. Therapeutic liposomal dry powder inhalation aerosols for targeted lung delivery. Lung. 2012;190:251-262. doi:10.1007/s00408-011-9360-x

121. Minko T, Stefanov A, Pozharov V. Selected contribution: lung hypoxia: antioxidant and antiapoptotic effects of liposomal $\alpha$ tocopherol. J Appl Physiol. 2002;93:1550-1560. doi:10.1152/ japplphysiol.00007.2002

122. Garbuzenko OB, Mainelis G, Taratula O, Minko T. Inhalation treatment of lung cancer: the influence of composition, size and shape of nanocarriers on their lung accumulation and retention. Cancer Biol Med. 2014;11:44-55. doi:10.7497/j.issn.20953941.2014.01.004

123. Ivanova V, Garbuzenko OB, Reuhl KR, et al. Inhalation treatment of pulmonary fibrosis by liposomal prostaglandin E2. Eur $J$ Pharm Biopharm. 2013;84:335-344. doi:10.1016/j.ejpb.2012. 11.023

124. Li N, Weng D, Wang S-M, et al. Surfactant protein-A nanobodyconjugated liposomes loaded with methylprednisolone increase lung-targeting specificity and therapeutic effect for acute lung injury. Drug Deliv. 2017;24:1770-1781. doi:10.1080/ 10717544.2017.1402217

125. Cipolla D, Blanchard J, Gonda I. Development of liposomal ciprofloxacin to treat lung infections. Pharmaceutics. 2016;8:6. doi:10.3390/pharmaceutics8010006

126. Meenach SA, Anderson KW, Hilt JZ, McGarry RC, Mansour HM. High-performing dry powder inhalers of paclitaxel DPPC/ DPPG lung surfactant-mimic multifunctional particles in lung cancer: physicochemical characterization, in vitro aerosol dispersion, and cellular studies. AAPS Pharm Sci Tech. 2014;15:15741587. doi:10.1208/s12249-014-0182-Z

127. Wang Y, Kho K, Cheow WS, Hadinoto K. A comparison between spray drying and spray freeze drying for dry powder inhaler formulation of drug-loaded lipid-polymer hybrid nanoparticles. Int J Pharm. 2012;424:98-106. doi:10.1016/j.ijpharm.2011. 12.045

128. Sinha B, Mukherjee B, Pattnaik G. Poly-lactide-co-glycolide nanoparticles containing voriconazole for pulmonary delivery: in vitro and in vivo study. Nanomedicine. 2013;9:94-104. doi:10.1016/j.nano.2012.04.005

129. Chen AM, Taratula O, Wei D, et al. Labile catalytic packaging of DNA/siRNA: control of gold nanoparticles "out" of DNA/siRNA complexes. ACS Nano. 2010;4:3679-3688. doi:10.1021/nn90 1796 n

130. Pitard B, Bello-Roufaï M, Lambert O, et al. Negatively charged self-assembling DNA/poloxamine nanospheres for in vivo gene transfer. Nucleic Acids Res. 2004;32:e159. doi:10.1093/nar/ gnh153

131. Tomoda K, Ohkoshi T, Hirota K, et al. Preparation and properties of inhalable nanocomposite particles for treatment of lung cancer. Colloids Surf B Biointerfaces. 2009;71:177-182. doi:10.1016/j. colsurfb.2009.02.001

132. Bivas-Benita M, Romeijn S, Junginger HE, Borchard G. PLGAPEI nanoparticles for gene delivery to pulmonary epithelium. Eur J Pharm Biopharm. 2004;58:1-6. doi:10.1016/j.ejpb.2004.03.008

133. Vij N. Nano-based theranostics for chronic obstructive lung diseases: challenges and therapeutic potential. Expert Opin Drug Deliv. 2011;8:1105-1109. doi:10.1517/17425247.2011.597381

134. Kaminskas LM, McLeod VM, Ryan GM, et al. Pulmonary administration of a doxorubicin-conjugated dendrimer enhances drug exposure to lung metastases and improves cancer therapy. $J$ Control Release. 2014;183:18-26. doi:10.1016/j.jconrel.2014. 03.012 
135. Patil ML, Zhang M, Betigeri S, et al. Surface-modified and internally cationic polyamidoamine dendrimers for efficient siRNA delivery. Bioconjug Chem. 2008;19:1396-1403. doi:10.1021/bc8000722

136. Khan OF, Zaia EW, Jhunjhunwala S, et al. Dendrimer-inspired nanomaterials for the in vivo delivery of siRNA to lung vasculature. Nano Lett. 2015;15:3008-3016. doi:10.1021/n15048972

137. Bohr A, Tsapis N, Andreana I, et al. Anti-inflammatory effect of anti-TNF- $\alpha$ SiRNA cationic phosphorus dendrimer nanocomplexes administered intranasally in a murine acute lung injury model. Biomacromolecules. 2017;18:2379-2388.

138. Jiang J, Liu Y, Wu C, et al. Development of drug-loaded chitosan hollow nanoparticles for delivery of paclitaxel to human lung cancer A549 cells. Drug Dev Ind Pharm. 2017;43:1304-1313. doi:10.1080/03639045.2017.1318895

139. Price DN, Stromberg LR, Kunda NK, Muttil P. In vivo pulmonary delivery and magnetic-targeting of dry powder nano-in-microparticles. Mol Pharm. 2017;14:4741-4750. doi:10.1021/acs. molpharmaceut.7b00532

140. Fytianos K, Chortarea S, Rodriguez-Lorenzo L, et al. Aerosol delivery of functionalized gold nanoparticles target and activate dendritic cells in a 3D lung cellular model. ACS Nano. 2017;11:375-383. doi:10.1021/acsnano.6b06061

141. Bhushan B, Gopinath P. Antioxidant nanozyme: a facile synthesis and evaluation of the reactive oxygen species scavenging potential of nanoceria encapsulated albumin nanoparticles. $J$ Mater Chem. 2015;B3:4843-4852. doi:10.1039/C5TB00572H

142. Muralidharan P, Hayes D, Black SM, Mansour HM. Microparticulate/nanoparticulate powders of a novel Nrf2 activator and an aerosol performance enhancer for pulmonary delivery targeting the lung Nrf2/Keap-1 pathway. Mol Syst Des Eng. 2016;1:48-65. doi:10.1039/C5ME00004A

143. Roulet A, Armand L, Dagouassat M, et al. Intratracheally administered titanium dioxide or carbon black nanoparticles do not aggravate elastase-induced pulmonary emphysema in rats. $B M C$ Pulm Med. 2012;12:38. doi:10.1186/1471-2466-12-38

144. Al Faraj A, Shaik AS, Afzal S, Al Sayed B, Halwani R. MR imaging and targeting of a specific alveolar macrophage subpopulation in LPS-induced COPD animal model using antibodyconjugated magnetic nanoparticles. Int $J$ Nanomedicine. 2014;9:1491-1503. doi:10.2147/IJN.S59394

145. Weng KC, Noble CO, Papahadjopoulos-Sternberg B, et al. Targeted tumor cell internalization and imaging of multifunctional quantum dot-conjugated immunoliposomes in vitro and in vivo. Nano Lett. 2008;8:2851-2857. doi:10.1021/nl801488u

146. Liong M, Lu J, Kovochich M, et al. Multifunctional inorganic nanoparticles for imaging, targeting, and drug delivery. ACS Nano. 2008;2:889-896. doi:10.1021/nn800072t

147. Al-Jamal WT, Kostarelos K. Liposomes: from a clinically established drug delivery system to a nanoparticle platform for theranostic nanomedicine. Acc Chem Res. 2011;44:1094-1104. doi:10.1021/ar200105p
148. Davis SS. Biomédical applications of nanotechnology — implications for drug targeting and gene therapy. Trends Biotechnol. 1997;15:217-224. doi:10.1016/S0167-7799(97)01036-6

149. Sahib MN, Darwis Y, Peh KK, Abdulameer SA, Tan YTF. Rehydrated sterically stabilized phospholipid nanomicelles of budesonide for nebulization: physicochemical characterization and in vitro, in vivo evaluations. Int J Nanomed. 2011;6:23512366. doi:10.2147/IJN.S25363

150. Monument MJ, Hart DA, Befus AD, et al. The mast cell stabilizer ketotifen reduces joint capsule fibrosis in a rabbit model of posttraumatic joint contractures. Inflamm Res. 2012;61:285-292. doi:10.1007/s00011-011-0409-3

151. Chen DL, Rosenbluth DB, Mintun MA, Schuster DP. FDG-PET imaging of pulmonary inflammation in healthy volunteers after airway instillation of endotoxin. J Appl Physiol. 2006;100:16021609. doi:10.1152/japplphysiol.01429.2005

152. Kubota R, Yamada S, Kubota K, et al. Intratumoral distribution of fluorine-18-fluorodeoxyglucose in vivo: high accumulation in macrophages and granulation tissues studied by microautoradiography. J Nucl Med. 1992;33:1972-1980.

153. Swaminathan J, Ehrhardt C. Liposomal delivery of proteins and peptides. Expert Opin Drug Deliv. 2012;9:1489-1503. doi:10.1517/17425247.2012.735658

154. Mueller C, Flotte TR. Gene therapy for cystic fibrosis. Clin Rev Allergy Immunol. 2008;35:164-178. doi:10.1007/s12016-008-8080-3

155. Al-Jamal R, Wallace W, Harrison D. Gene therapy for chronic obstructive pulmonary disease: twilight or triumph? Expert Opin Biol Ther. 2005;5:333-346. doi:10.1517/14712598.5.3.333

156. Stecenko AA, Brigham KL. Gene therapy progress and prospects: alpha-1 antitrypsin. Gene Ther. 2003;10:95-99. doi:10.1038/sj. gt.3301947

157. Metz R, DiCola M, Kurihara T, et al. Mode of action of RNA/ DNA oligonucleotides: progress in the development of gene repair as a therapy for alpha(1)-antitrypsin deficiency. Chest. 2002;121:91S-97S. doi:10.1378/chest.121.3_suppl.91S

158. Liu A, Liu L, Chen S, et al. Activation of canonical wnt pathway promotes differentiation of mouse bone marrow-derived MSCs into type II alveolar epithelial cells, confers resistance to oxidative stress, and promotes their migration to injured lung tissue in vitro. $J$ Cell Physiol. 2013;228:1270-1283. doi:10.1002/jcp.24282

159. Zhao Y, Xu A, Xu Q, et al. Bone marrow mesenchymal stem cell transplantation for treatment of emphysemic rats. Int J Clin Exp Med. 2014;7:968-972.

160. Rojas M, Xu J, Woods CR, et al. Bone marrow-derived mesenchymal stem cells in repair of the injured lung. Am J Respir Cell Mol Biol. 2005;33:145-152. doi:10.1165/rcmb.2004-0330OC
International Journal of Nanomedicine

\section{Publish your work in this journal}

The International Journal of Nanomedicine is an international, peerreviewed journal focusing on the application of nanotechnology in diagnostics, therapeutics, and drug delivery systems throughout the biomedical field. This journal is indexed on PubMed Central, MedLine, CAS, SciSearch ${ }^{\mathbb{}}$, Current Contents ${ }^{\mathbb{R}} /$ Clinical Medicine,
Journal Citation Reports/Science Edition, EMBase, Scopus and the Elsevier Bibliographic databases. The manuscript management system is completely online and includes a very quick and fair peer-review system, which is all easy to use. Visit http://www.dovepress.com/ testimonials.php to read real quotes from published authors. 